\title{
A genetic approach to dissect the role of prefoldins in Arabidopsis
}

Noel Blanco-Touriñán ${ }^{1,4}$, David Esteve-Bruna ${ }^{1,4}$, Antonio Serrano-Mislata ${ }^{1}$, Rosa María Esquinas ${ }^{1}$, Francesca Resentini ${ }^{2}$, Javier Forment ${ }^{1}$, Cristian Carrasco-López ${ }^{3}$, Claudio Novella-Rausell ${ }^{1}$, Alberto Palacios ${ }^{1}$, Pedro Carrasco ${ }^{4}$, Julio Salinas ${ }^{3}$, Miguel Á. Blázquez ${ }^{1}$, David Alabadí ${ }^{1 *}$

${ }^{1}$ Instituto de Biología Molecular y Celular de Plantas (CSIC-Universidad Politécnica de Valencia), 46022 Valencia, Spain

2Dipartimento di Bioscienze, Università degli Studi di Milano, 20133 Milano, Italy

${ }^{3}$ Departamento de Biotecnología Microbiana y de Plantas, Centro de Investigaciones Biológicas (CSIC), 28040 Madrid, Spain

${ }^{4}$ Departament de Bioquímica i Biologia Molecular, Universitat de València, 46100

Burjassot, Spain

"Corresponding author: David Alabadí (dalabadi@ibmcp.upv.es; phone: +34963877723)

${ }^{4}$ These authors contributed equally to this work. 


\section{Summary}

The prefoldin complex (PFDc) was identified in humans as co-chaperone of the cytosolic chaperonin TRiC/CCT. It is conserved in eukaryotes and is composed of subunits PFD1 to 6. PFDc-TRiC/CCT operates folding actin and tubulins. In addition to this function, PFDs participate in a wide range of cellular processes, both in the cytoplasm and in the nucleus, and their malfunction cause developmental alterations and disease in animals, and altered growth and environmental responses in yeast and plants. Genetic analyses in yeast indicate that not all functions performed by PFDs require the participation of the canonical complex. The lack of systematic genetic analyses in higher eukaryotes makes it difficult to discern whether PFDs participate in a particular process as canonical complex or in alternative configurations, i.e. as individual subunits or in other complexes. To tackle this question, and on the premise that the canonical complex cannot be formed if one subunit is missing, we have prepared an Arabidopsis mutant deficient in the six prefoldins, and compared various growth and environmental responses with those of the individual $p f d$. In this way, we demonstrate that the PFDc is required to delay flowering, for seed germination, or to respond to high salt stress, whereas two or more PFDs redundantly attenuate the response to osmotic stress. A coexpression analysis of differentially expressed genes in the sextuple mutant has identified several transcription factors, such as ABI5 or PIF4, acting downstream of PFDs. Furthermore, it has made possible to assign novel roles for PFDs, for instance, in the response to warm temperature.

\section{Keywords}

prefoldin complex, co-chaperone, $\mathrm{TRiC/CCT}$, flowering time, stress, temperature, auxin 


\section{Introduction}

Prefoldins (PFDs) are conserved proteins present in archaea and in eukaryotes that were identified in humans and in yeast as part of a hexameric complex, called PFD complex (PFDc; Vainberg et al., 1998; Geissler et al., 1998). PFDs can be classified into $\alpha$ - or $\beta$-type depending on their structure (Figure S1a) (Arranz et al., 2018). In eukaryotes there are two $\alpha$-type (PFD3 and PFD5) and four $\beta$-type (PFD1, PFD2, PFD4, and PFD6) PFDs, whereas in archaea only one PFD per type is found. The PFDc adopts a jellyfishlike structure in which two $\alpha$-subunits occupy a central position allowing the binding of four $\beta$-subunits (Siegert et al., 2000; Martin-Benito et al., 2002). In eukaryotes, the arrangement of the different subunits within the complex appears to be conserved (Gestaut et al., 2019).

Currently, the best characterized function of the PFDc is in proteostasis, as cochaperone of the chaperonin TRiC/CCT in the folding of tubulins and actin (Gestaut et al., 2019). Authors show that the substrate protein is transferred between the active sites of PFDc and TRiC/CCT until it is properly folded, avoiding the formation of deleterious protein aggregates. Yeast gim/pfd mutants show very similar cytoskeleton-related phenotypes, such as reduced $\alpha$-tubulin levels (Vainberg et al., 1998; Geissler et al., 1998), which are not aggravated when several gim/pfd mutations are combined (Siegers et al., 1999). Tubulin- and actin-related phenotypes are also observed in pfd mutants in other model organisms. A missense mutation in the PFDN5 gene causes developmental alterations in the central nervous system in mice, which are associated to reduced accumulation of $\alpha$-tubulin and $\beta$-actin (Lee et al., 2011). Hypomorphic alleles of the Drosophila MGR locus, which encodes PFD3, cause defects in the formation of the meiotic spindle due to reduced tubulin levels, being this reduction also observed in fly DMEL-2 cells after knocking down PFD4 (Delgehyr et al., 2012). Knock down of PFD genes in C. elegans, except PFD4 that is divergent in this species, causes impaired cell division and embryo lethality due to defects in the rate of microtubule polymerization (Lundin et al., 2008). In Arabidopsis, pfd mutations provoke defects in the arrangement of cortical microtubules (MT) and in the formation of the phragmoplast, leading to impaired cell elongation and division, respectively (Gu et al., 2008; Rodriguez-Milla and Salinas, 2009; Perea-Resa et al., 2017). In summary, the similar phenotypes caused by mutations in individual PFD genes is consistent with the idea that the function of the PFDc is impaired when a subunit is missing. This view is further supported by the unique arrangement of subunits within the complex, based on specific protein-protein interactions (Gestaut et al., 2019).

Nevertheless, genetic analyses in yeast have shown that other functions of PFDs are not performed by the canonical PFDc. All PFDs, except GIM2/PFD3 and GIM4/PFD2, are required for transcription elongation of long genes and bind chromatin in a transcription-dependent manner (Millan-Zambrano et al., 2013). Furthermore, no additivity was found when combining affected gim/pfd mutants, which suggested that these PFDs may exert this role by being part of an alternative complex. In the same line, only GIM2/PFD3, GIM3/PFD4, and GIM1/PFD6 proteins are required for the transcription of genes in response to osmotic or oxidative stress (Amorim et al., 2017).

The implication of PFDs in other cellular processes is well documented (Liang et al., 2020), however, the lack of systematic genetic analyses makes it difficult to discern whether a particular role is exerted by the canonical PFDc or by individual subunits. For example, PFDN5/MM-1 acts as bridge protein that recruits a corepressor complex to the c-Myc transcription factor (Satou et al., 2001). Although the participation of PFDN5/MM1 as c-Myc partner is demonstrated genetically (Fujioka et al., 2001) and the experimental data suggest that PFDN5/MM-1 fulfills this function, it cannot be ruled out that this role is performed as part of the PFDc. In Arabidopsis, PFD4 promotes the proteasomal degradation of the transcription factor HY5, and accordingly, its levels are 
augmented in pfd4 mutants (Perea-Resa et al., 2017). HY5 levels are also increased in pfd3 and in pfd5 mutants, indicating that these other subunits are also involved. However, the limited genetic analysis precludes clarification as to whether this is a role performed by the canonical complex.

PFDs participate in diverse cellular processes in eukaryotes and their impaired function leads to disease and developmental abnormalities in animals (Liang et al., 2020), and to altered growth and response to environmental cues in plants (Rodriguez-Milla and Salinas, 2009; Perea-Resa et al., 2017; Esteve-Bruna et al., 2020) and in yeast (MillanZambrano et al., 2013; Siegers et al., 1999; Amorim et al., 2017). To understand the roles of PFDs in cellular processes, one of the issues that we need to clarify is whether they act as a canonical PFDc or as individual subunits in each case. To address this question in Arabidopsis, and since the function of the complex is impaired if one subunit is missing, we have prepared a mutant defective in the six PFDs and compared its growth habit and its behavior under various stresses with those of the individual mutants. Furthermore, we have identified novel functions for PFDs based on a transcriptomic analysis of the sextuple mutant.

\section{Results}

\section{The PFDc forms in vivo}

We first investigated whether the Arabidopsis PFDs can adopt the structure of their orthologs in yeast and humans. The structure of the Arabidopsis PFDs could be modeled in silico based on the structure of their human orthologs (Figure S1a) and assembled to form the jellyfish-like complex (Gestaut et al., 2019) (see the top view in Figure 1a and the comparison of the two complexes in Figure S1b). The high similarity between complexes suggests that the PFDc would adopt the same 3D arrangement in Arabidopsis and in humans in vivo.

We next determined if the PFD proteins associate in vivo. For that purpose, we performed Tandem Affinity Purification using an Arabidopsis PSB-D cell suspension line expressing the GS-PFD3 fusion protein. After the two sequential immunopurification steps, the top PFD3 interactors identified in two replicates were the other five PFDs (Figure 1b), suggesting that the PFDc forms in vivo. Indeed, when we subjected extracts of seedlings expressing either PFD4pro::PFD4-GFP (Perea-Resa et al., 2017) or 35Spro::PFD6-YFP (Esteve-Bruna et al., 2020) to gel filtration, the elution profiles of both proteins indicated that they may be incorporated into protein complexes of molecular weight compatible with the PFDc (ca. $130 \mathrm{KDa}$ including the fusion protein) (Figure 1c).

\section{Preparation of the $6 x$ pfd mutant}

In order to investigate the PFDs' contribution to Arabidopsis development and response to the environment, and to determine in which cases PFDs participate as complex, we set out to prepare a sextuple mutant defective in the activity of the six PFDs. Mutants for PFD genes have been described in Arabidopsis, except for PFD1 (Gu et al., 2008; Rodriguez-Milla and Salinas, 2009; Esteve-Bruna et al., 2020; Perea-Resa et al., 2017). We identified a T-DNA mutant for the PFD1 gene in the GABI-Kat collection (Kleinboelting et al., 2012). The pfd1 mutant carries the T-DNA inserted in the third exon (Figure S2a) and is null, or highly hypomorphic, as evidenced by the inability to amplify the full-length transcript by RT-PCR (Figure S2b). With available mutants for all PFD genes, we prepared the pfd1 pfd2 pfd3 pfd4 pfd5 pfd6-1 sextuple mutant (hereafter referred to as $6 x \mathrm{pfd}$ ) by genetic crosses (see Methods section for details). An RNA-seq analysis of the sextuple mutant (see below) confirmed that $p f d 1, p f d 2, p f d 3$, and $p f d 4$ alleles are null or highly hypomorphic and that pfd6-1 carries the reported point mutation (Figure S3a). Nonetheless, it also showed that the PFD5 gene was transcribed in the mutant, albeit at a reduced level (ca. $40 \%$ of wild type) (Figure S3a and S3b). This result contrasts with the absence of full-length PFD5 transcript previously reported in the $p f d 5$ 
mutant (Rodriguez-Milla and Salinas, 2009). The insertion site is in the third intron (Figure S3c), suggesting that the T-DNA may be processed in a fraction of PFD5 premRNAs and that this occurs more often in the sextuple mutant than in the pfd5. After having obtained the $6 x \mathrm{pfd}$ mutant, we investigated various growth and environmental responses in this mutant and compared them with those in individual pfd.

\section{PFDs participate in microtubule organization exclusively as canonical complex}

Defects in the organization of MT have been described in Arabidopsis for the pfd3, pfd4, pfd5, and pfd6 mutants (Gu et al., 2008; Rodriguez-Milla and Salinas, 2009; Perea-Resa et al., 2017). To determine if pfd1 and pfd 2 mutations also cause defects in the MT organization, we introduced the microtubule marker UBQ10::Venus-TUA6 (Salanenka et al., 2018) into both mutant backgrounds by genetic crosses. We imaged MT by confocal microscopy in two populations of cells in 3-day-old etiolated seedlings, apical hook cells, which have ceased elongation and have disorganized, randomly arranged MT, and in cells just below the apical hook, which undergo elongation and have organized MT, arranged parallel to the growth axis (Gu et al., 2008). MT were disorganized in apical hook cells in the wild type and organized in elongating cells below the hook (Figure 2a). The same MT organization was observed in the pfd1 mutant, while MT were randomly arranged in both types of cells in pfd2 seedlings. We analyzed other phenotypes dependent on tubulin folding: (i) the sensitivity to the microtubule-depolymerizing drug oryzalin and (ii) tubulin levels. pfd1 seedlings showed similar response to oryzalin than the other pfd mutants, including $p f d 2$, although reduced sensitivity was observed at the lowest concentration (Figure 2b). $\alpha$-tubulin levels were reduced in all mutants (Figure 2c and d). In summary, these results show a similar behavior for all pfd mutants regarding microtubule-related phenotypes. The lack of apparent defects in the MT organization in the pfd 1 mutant may be due to the lower sensitivity of this assay to PFD1 deficiency compared to the other two.

We next investigated if this role is carried out by the PFDc. Studies in yeast and in humans indicate that it is involved (Gestaut et al., 2019). Nonetheless, the lack of genetic analyses makes it difficult to rule out a complex-independent role for the individual subunits. To tackle this question, we compared phenotypes of the $6 x$ pfd mutant with those of the individual mutants. On the premise that the complex cannot be formed if a subunit is missing, the rationale is that the microtubule-related phenotypes of individual pfd and the $6 x$ pfd mutants would be the same if the role of PFDs is performed entirely by the PFDc. The sextuple mutant showed the same sensitivity to oryzalin and $\alpha$-tubulin levels than the individual pfd (Figure 1b-d). These results are the genetic demonstration that PFDs participate in microtubule-related processes exclusively as part of the PFDc.

\section{Complex-dependent and -independent contributions of PFDs to organ growth}

Reduced growth is a common trait of pfd mutants (Perea-Resa et al., 2017; RodriguezMilla and Salinas, 2009; Esteve-Bruna et al., 2020; Gu et al., 2008). We next sought to determine if PFDs' contribution to organ growth is mediated by the PFDc. We analyzed the size of the rosette and hypocotyl and root length in individual pfd mutants and in the $6 x \mathrm{pfd}$. The rosette size was reduced to a similar extent in individual pfd mutants and further reduced in the $6 x$ pfd (Figures $3 a$ and $b$ and S4). Microtubule-related defects leading to altered cell division and/or expansion may contribute to rosette growth alterations in individual pfd mutants. Nonetheless, the fact that the sextuple mutant exhibits a further reduction in size indicates that other processes, non-related to microtubules and controlled redundantly by two or more PFDs, are also altered in this mutant. PFDs therefore contribute to the rosette growth in two ways, dependent and independent of the PFDc.

The analysis of the hypocotyl and root length revealed a similar reduction in the size of both organs in individual pfd mutants and in the sextuple (Figure $3 c$ and d). Despite the 
slight but significant differences among genotypes, the lack of an additive effect in the 6x pfd mutant suggests that PFDs act as complex to promote both hypocotyl and root growth. At least part of the contribution of the PFDc to hypocotyl elongation may be mediated by its role in MT organization, since the growth of this organ is almost entirely mediated by cell expansion (Gendreau et al., 1997). In addition to cell expansion, cell divisions occurring in the root meristem also contribute to the growth of this organ. Therefore, PFDs contribute to root growth as complex that may mediate, at least, microtubule-dependent cell division and cell elongation.

\section{The PFDc contributes to the regulation of flowering time}

We reasoned that the increased expression of PFD genes in the vegetative rosette and in the shoot apex, before and after the transition to flowering, would be compatible with a role for PFDs in flowering time regulation (Figure S5) (Winter et al., 2007). To test this hypothesis and to determine eventually whether this role is performed by the PFDc, we measured the flowering time of all individual pfd mutants and of the sextuple grown in short days (SD). Results show that all mutants flowered earlier than the wild type (Figure 4a). The phenotype was similar for all mutant lines, albeit the effect of the pfd1 mutation was milder. Importantly, the absence of additive effects in the $6 x$ pfd suggests that the activity of PFDs on flowering time is exerted by the PFDc. This effect is independent of the photoperiod, since early flowering was also observed when the $6 x$ pfd mutant was grown under long days (LD) (Figures 4b and S6a).

Next, to try understanding how the PFDc contributes to the flowering time, we investigated whether the expression of key regulatory genes is altered in the $6 x$ pfd mutant (Figure 4c). The analysis included SPL9 and SPL15 (aging pathway); FLM, SVP, and $F L C$ (vernalization and autonomous pathways); $G I$ and $C O$ (photoperiod pathway); GA20ox2 and GA2ox2 (gibberellin pathway); FT, FD, TSF, BFT, and SOC1 (integrator genes); and $L F Y$ and $A P 1$ (meristem identity genes) (Fornara et al., 2010). We analyzed their expression by RT-qPCR in shoot apexes and/or in the second oldest rosette leaf from 14-day-old plants grown in LD. Among representative genes of different pathways, only the expression of GA2ox2 was altered in the mutant (Figures $4 \mathrm{~d}$ and S6b and c). We identified, nonetheless, the FT-TSF module as the main target of the PFDc. The expression of $F T$ in leaves and of TSF in the shoot apex was higher in the sextuple mutant than in the wild type, which would explain the higher transcript levels of the downstream genes SOC1, LFY, and AP1 that lead to the floral transition (Figures $4 \mathrm{~d}$ and $\mathrm{e}$ and $\mathrm{S} 6 \mathrm{~b}$ and $\mathrm{c}$ ). These results suggest that the early flowering of the $6 x$ pfd mutant is associated to increased FT-TSF activity. The PFDc, therefore, is required to delay flowering by repressing the expression of integrator genes.

\section{Individual PFDs attenuate the cold acclimation response}

We next investigated the behavior of the $6 x \mathrm{pfd}$ and individual pfd mutants when exposed to environmental challenges. PFD3, PFD4, and PFD5 attenuate the acclimation to low temperatures (Perea-Resa et al., 2017). The individual pfd 1 and pfd 2 mutants showed the same freezing tolerance as $p f d 3, p f d 4$, and $p f d 5$, whereas a wild-type response was observed for pfd6-1 (Figure $5 a$ and $b$ ). The $6 x$ pfd plants showed a similar behavior than pfd1 to pfd5. Taken together, our findings suggest that (i) PFD6 is not essential in controlling the adaptive response to low temperatures, and (ii) PFDs do not participate as canonical complex in this response. Nonetheless, since the pfd6-1 allele carries a missense mutation that does not appear to interfere with the in silico assembly of the PFDc (Figure 5c), an alternative possibility is that PFDs participate in the low temperature response as canonical complex, and that the pfd6-1 mutation does not affect the contribution of PFD6 to the function of the complex in this process, contrary to what occurs in others (Figures 2-4). 
bioRxiv preprint doi: https://doi.org/10.1101/2021.01.28.428673; this version posted January $29,2021$. The copyright holder for this preprint (which was not certified by peer review) is the author/funder, who has granted bioRxiv a license to display the preprint in perpetuity. It is made available under aCC-BY-NC-ND 4.0 International license.

\section{Different contributions of PFDs to the response to salt and osmotic stress}

The activity of PFD3, PFD4, and PFD5 is required for the plant's response to salt stress (Rodriguez-Milla and Salinas, 2009; Esteve-Bruna et al., 2020). The root growth of pfd2 and pfd6-1 mutants was affected by the $100 \mathrm{mM} \mathrm{NaCl}$ treatment in a similar way to that of the pfd3, pfd4, or pfd5 mutants, while it was less affected in the pfd1 (Figure 6a). Interestingly, 6x pfd seedlings behaved as the individual pfd, suggesting that PFDs contribute to the response to high salt as PFDc.

The hypersensitive response to $\mathrm{NaCl}$ could be caused by either the ionic or the osmotic component of the treatment. Previous findings show that the sensitivity of $p f d 3$ and $p f d 5$ mutants is likely $\mathrm{Na}^{+}$-specific, because they are not hypersensitive to $\mathrm{LiCl}$ or mannitol (Rodriguez-Milla and Salinas, 2009). Nevertheless, it cannot be ruled out a general effect on ionic or osmotic stress if some PFDs act redundantly. We measured the root length of individual pfd and the $6 x$ pfd mutants in the presence of $12 \mathrm{mM} \mathrm{LiCl}$ or $300 \mathrm{mM}$ mannitol. LiCl stress inhibited root growth in a similar way in all genotypes, ruling out any involvement of PFDs in the response to nonspecific ionic stress (Figure 6b). Interestingly, the $6 x$ pfd seedlings were more tolerant to the mannitol treatment, whereas a wild-type response was observed for individual pfd mutants (Figure 6c). These results indicate that the response of the plant to osmotic stress does not require the participation of the PFDc, but rather two or more PFD subunits that redundantly attenuate the response. Moreover, this result also indicates that it is unlikely that there is contribution of osmotic stress to the effect of high $\mathrm{NaCl}$ in pfd mutants.

The participation of PFDs in cold acclimation is reflected in augmented levels of PFD4 transcript and protein in response to low temperatures (Perea-Resa et al., 2017). We investigated whether PFDs are subject to transcriptional or post-transcriptional regulation in response to salt or osmotic stress. Neither the expression of PFD genes nor levels of PFD4-GFP and PFD6-YFP proteins were affected by treatments with $\mathrm{NaCl}$ or mannitol, contrary to control genes or proteins (Figures S7 and S8).

\section{PFDs mostly contribute to gene expression independently of the PFDc}

Impaired activity of PFDs results in altered gene expression (Esteve-Bruna et al., 2020). To determine whether PFDs participate in gene expression as complex, we compared the differential expressed genes (DEGs) identified by RNA-seq in the pfd4 and $6 x$ pfd mutants. A total of 1186 DEGs, 734 up- and 452 down-regulated, were identified in the $6 x$ pfd mutant ( $\left[\log _{2} \mathrm{FC}\right] \geq 2, P<0.05$; Figure $7 \mathrm{a}$ and $\mathrm{b}$ and Supplementary File 1$)$. The relatively high number of misexpressed genes in the mutant highlights the relevance of PFDs' activity for gene expression.

The number of DEGs was significantly lower in the pfd4 mutant (198 DEGs, $\left[\log _{2} \mathrm{FC}\right] \geq$ 2, $P<0.05$; Figure 7a and $\mathrm{b}$ and Supplementary File 2). A lower number of DEGs was also observed when the pfd 4 mutant was grown in soil instead of in vitro (117 DEGs, $\left[\log _{2} \mathrm{FC}\right] \geq 2, P<0.05$; Supplementary File 2; Esteve-Bruna et al., 2020). The fact that the molecular phenotype is more severe in the $6 x$ pfd mutant than in the pfd4 suggests that the contribution of individual PFDs to gene expression is greater than that of the PFDc. It is important to note, however, that the majority of DEGs in pfd4 seedlings, grown either in vitro or in soil, were not misexpressed in the 6x pfd mutant (Figure 7b). Among the common genes, only the upregulated ones preferably followed the same trend (Figures $7 \mathrm{~b}$ and $\mathrm{S} 9 \mathrm{a}$ ). The fact that the effect of the pfd 4 mutation on gene expression is less when found in a cellular context with mutations in all other PFD genes, suggests that part of the effect in the pfd4 mutant may be due to overaccumulation of other PFDs.

\section{PFDs act upstream of a few TFs}

We next wondered how PFDs affect gene expression. They may act via multiple pathways, but they may also act through few transcription factors (TFs) that control most 
of the PFD-dependent transcriptome. We reasoned that we could infer their behavior by looking at the architecture of the coexpression network of DEGs, which would likely organize itself into compact gene clusters if PFDs act through few TFs. We used the tool CORNET 3.0 (De Bodt et al., 2012) to determine the pairwise coexpression values among the 1186 DEGs in the $6 x$ pfd mutant in 454 microarray experiments. The analysis resulted in a network with 636 nodes and 9646 edges (Supplementary File 3), mostly organized into three compact gene clusters (Figure 7c). Interestingly, cluster 1 was exclusively formed by upregulated genes, while the two others mostly included downregulated genes (Supplementary File 4). The highest coexpression values were observed between genes of the same cluster (see Figure S9b for a larger image of clusters). This organization would be consistent with genes in each cluster being controlled by few TFs.

To investigate this possibility, we used the TF2Network tool that allows to identify putative regulatory TFs based in coexpression and DNA binding data (Kulkarni et al., 2018). We found that $80 \%$ of genes in cluster 1 are coexpressed with $A B / 5$ and $69 \%$ of them are direct targets of this TF. Importantly, $A B I 5$ itself was among the upregulated genes in the $6 x$ pfd mutant (Figure $7 \mathrm{~d}$ and Supplementary File 1). ABI5 is a bZIP TF that plays a positive role in ABA signaling (Skubacz et al., 2016), and, in agreement with this, a GO analysis showed that the category "response to ABA stimulus" was overrepresented in cluster $1\left(P=1.5 \times 10^{-11}\right)$. Between $39 \%$ and $63 \%$ of downregulated genes of cluster 2 were coexpressed with AtWRKY TFs (AtWRKY65, 36, 72, 35, 29, 9, and 59; cited from the most to the least coexpressed). AtWRKY72 was also misexpressed in the mutant (Figure $7 \mathrm{~d}$ and Supplementary File 1). The latter and AtWRKY29 have been characterized, being related to defense against pathogens (Bhattarai et al., 2010; Zhou et al., 2004). The analysis of cluster 3 showed poor coexpression values for any TF (less than 17\%). Nonetheless, it revealed that $66 \%$ and $33 \%$ of genes were direct targets of GBF3 and PIF4, respectively. These TFs were also misexpressed in the $6 x$ pfd mutant (Figure $7 d$ and Supplementary File 1). GBF3 is a bZIP TF that contributes to the plant's response to abiotic stress (Ramegowda et al., 2017), and PIF4 is a well characterized bHLH TF that transmits information about ambient light and temperature to hormonal and growth pathways (Choi and Oh, 2016). In summary, the in silico analyses predicted that part of misregulated genes in the $6 x$ pfd mutant may be regulated by ABI5, WRKY72, GBF3, and PIF4.

\section{The transcriptome of the $\mathbf{6 x}$ pfd mutant reveals novel functions for PFDs}

To identify novel functions for PFDs, we searched for enriched Gene Ontology (GO) categories in the DEGs of $6 x$ pfd seedlings (Figure S9c, Supplementary File 1). The GO category "response to auxin stimulus" was more enriched in cluster $3\left(P=3.0 \times 10^{-9}\right)$ than when all DEGs were used $\left(P=2.1 \times 10^{-8}\right)$. Particularly striking is the presence of 13 auxin-responsive SAUR genes (Stortenbeker and Bemer, 2019) among those downregulated in cluster 3 (marked with an asterisk in Figure 8a). Indeed, another 13 SAUR together with the auxin biosynthesis gene YUC8 and the auxin signaling gene $I A A 29$, not represented in the microarray compendium used for coexpression analyses, also appeared downregulated in the 6x pfd mutant (Figure 8a and Supplementary File 1). These results suggest that impairment of PFDs' activity affects the auxin pathway.

The TF2Network analysis revealed that PIF4 probably controls the expression of genes in cluster 3. PIF4 is important for the expression of auxin biosynthesis and signaling genes, especially in response to warm temperature (Quint et al., 2016). In agreement with this, the enrichment of the GO category "response to auxin stimulus" was most significant among the direct targets of PIF4 in this cluster $\left(P=1.2 \times 10^{-15}\right)$. The reduced expression of PIF4 in the 6xpfd mutant may contribute to the low expression of the auxin genes. Nonetheless, we reasoned that the consequences of low PIF4 expression would be most obvious if we expose the $6 x p f d$ mutant to warm temperature. To test this, we 
selected five genes whose induction is PIF4-dependent: YUC8 (Sun et al., 2012), IAA19 (Huai et al., 2018), IAA29 (Koini et al., 2009), and SAUR19 and 23 (Franklin et al., 2011). IAA19 was included in the analysis although its expression was not altered in the $6 x$ pfd mutant. The RT-qPCR analysis confirmed both the results of the RNA-seq and the thermal induction of genes (Figure 8b). Notably, the induction at $29^{\circ} \mathrm{C}$ was mostly impaired for all genes in the mutant, except for SAUR19. These results indicate that PFDs are required for the proper response of seedlings to warm temperature. The fact that SAUR19 still responds to the temperature shift, despite it is dependent on PIF4 (Franklin et al., 2011), suggests that PFDs may affect the temperature response through other pathways in addition to those involving this transcription factor. The contribution of PFDs to the temperature response is not mediated by transcriptional regulation of PFD genes (Figure S10).

Since all PFD genes are transcriptionally active in imbibed seeds (Figure S5) and GO terms related to seed dormancy and germination were enriched in the DEGs in the $6 x$ pfd mutant (Figure S9c and Supplementary File 1), we wondered whether PFDs have a role in these processes. To determine the degree of dormancy and the germination capacity of $6 x$ pfd seeds, we compared their germination rate with that of the wild type with or without stratification at $4^{\circ} \mathrm{C}$ for $72 \mathrm{~h}$. Seeds of both genotypes harvested at the same time were used. The $6 x$ pfd seeds exhibited an enhanced degree of dormancy compared with the wild type (Figure 8c). Furthermore, mutant seeds showed a delay in germination after stratification (Figure $8 \mathrm{~d}$ ). The role of PFDs in germination might depend on the PFDc because seeds of all individual pfd mutants showed a delay in germination similar to that of $6 x$ pfd seeds (Figure S11).

\section{The $6 \times$ pfd mutant mimics transcriptional changes of wild-type plants exposed to stress}

The enrichment of GO categories related to abiotic stress among DEGs in the $6 x p f d$ mutant (Figure S9c and Supplementary File 1) suggests that it may constitutively manifest transcriptomic stress features. To investigate this possibility, we compared the transcriptome of $6 x$ pfd seedlings with that of wild-type seedlings exposed to either 150 $\mathrm{mM} \mathrm{NaCl}$ or $4{ }^{\circ} \mathrm{C}$ for $24 \mathrm{~h}$ (Esteve-Bruna et al., 2020). The metaanalysis revealed that $56.7 \%$ of the DEGs in the mutant were altered in the wild type exposed to either of the two stresses (Figures $9 a$ and Supplementary File 5), and that many of them behaved similarly (Figures 9b and S12). GO categories over-represented in the 163 DEGs common to the three conditions included response to abiotic stimuli and response to hormones (Figure 9c and Supplementary File 5). This indicates that PFDs are required to maintain adequate expression levels of many stress genes under non-stressful conditions.

Next, we hypothesized that PFDs are also required to achieve correct levels of gene expression in response to stress. To test this, we investigated the transcriptomic response of pfd 4 mutant seedlings exposed to either $150 \mathrm{mM} \mathrm{NaCl}$ or $4{ }^{\circ} \mathrm{C}$ for $24 \mathrm{~h}$. We found 2767 and 3718 DEGs in the pfd4 mutant after salt and the low temperature treatments, respectively (Figure S13 and Supplementary File 6). Although many genes were common to the wild type, a clear pfd4 signature was observed, since 1096 and 1121 DEGs were exclusively misexpressed in the mutant. These results indicate that at least PFD4 is required to reach proper expression level of many genes after the plant is subjected to stress.

\section{Discussion}

PFDs are required for normal development of animals and their malfunction, due to mutation or misexpression, is normally associated to cancer or disease (Liang et al., 2020) or causes embryo lethality (Lundin et al., 2008; Delgehyr et al., 2012). Arabidopsis pfd mutants, including the $6 x$ pfd, are viable and do not show apparent developmental 
defects beyond reduced size, indicating that PFDs' activity is mostly dispensable for normal development in this species (this work; Esteve-Bruna et al., 2020; RodriguezMilla and Salinas, 2009; Perea-Resa et al., 2017; Gu et al., 2008). Rather, their role in plants appears to be more relevant for properly interpreting environmental challenges (Esteve-Bruna et al., 2020; Rodriguez-Milla and Salinas, 2009; Perea-Resa et al., 2017). In fact, we show here that plants defective in the six PFDs perform better than the wild type when exposed to osmotic stress, which adds to known defects in the response to high salt or low temperature. It is important to note that our results expand this view. We show that they are not only required to respond to stress, but also to respond to environmental changes that in principle do not represent a stressful scenario for the plant, such as a moderate increase in the ambient temperature. This is further supported by the finding of enriched GOs related to environmental and stress responses in the gene set misregulated in the $6 x$ pfd mutant, and with the fact that the TFs identified by coexpression analyses acting downstream of PFDs mainly participate in environmental responses. The different effect of PFDs' malfunction on the plant's response to environmental changes, i.e. hypo- or hypersensitivity, is probably an indication of the varied modes of action through which these proteins act, which are just beginning to glimpse.

Determining whether PFDs participate in a particular process as members of the canonical PFDc or, conversely, in alternative configurations, i.e. other complexes or as individual subunits, provides clues to their mode of action. The analysis of the $6 x \mathrm{pfd}$ mutant has allowed us to identify processes that are dependent and independent of the activity of the canonical PFDc. Joint action with the TRiC/CCT would be expected for the PFDc in those processes that require the participation of the canonical complex (Gestaut et al., 2019). We have found that several growth-involving processes depend on it, in which it probably participates through the folding of at least tubulins. For other processes also depending on the PFDc, such as the plant's response to salt stress or the regulation of flowering time, the mechanistic connection with tubulins is not so obvious. Interestingly, altering microtubule polymerization affects gene expression in plants (Sangwan et al., 2001), and actin, another bona fide substrate of PFDc-TRiC/CCT, performs roles in the nucleus related to transcription (Blettinger et al., 2004). This opens up the possibility that the effect of the PFDc on the expression of the flowering integrator genes or of genes involved in salt stress is mediated through these two protein substrates. Alternatively, the PFDc may act through actin-related proteins, some of which are subunits of chromatin remodeling complexes that regulate the expression of $F T$ (Kumar et al., 2012; March-Diaz and Reyes, 2009) and also of stress genes (Wang et al., 2019). In fact, an actin-related protein, in this case belonging to a cytosolic complex, is substrate for TRiC/CCT chaperonin in vertebrates (Melki et al., 1993).

PFDc-TRiC/CCT may fold substrates other than tubulins or actin that could also mediate the function of the PFDc. Although not supported by functional or genetic analyses, interactomic approaches suggest that PFDc-TRiC/CCT assist the folding of the histone deacetylase HDAC1 in the nucleus of human cells (Banks et al., 2018). Similar approaches have identified the PFDc and TRiC/CCT complexes associated to the TOR kinase complex in Arabidopsis (Van Leene et al., 2019). In Drosophila, TRiC/CCT interacts physically with members of the TOR complex and is required for TOR function, probably by participating in its assembly (Kim and Choi, 2019). That the TOR complex is substrate of the PFDc-TRiC/CCT in plants is an interesting possibility that awaits investigation.

We have identified other processes in which the action of PFDs does not involve the canonical complex. Genetic analyses indicate that two or more PFDs act redundantly to promote rosette growth or to attenuate the response to osmotic stress. In these cases, it is more difficult to anticipate their mode of action. They may participate as individual 
subunits or as part of alternative complexes. For example, our previous results show that PFDs can participate in proteostasis independently of the PFDc. PFD4 acts as adaptor to mediate the stabilization of the spliceosome complex LSM2-8 by the chaperone Hsp90, a process in which PFD2 does not appear to be involved (Esteve-Bruna et al., 2020). Their participation in alternative complexes is a very exciting possibility that has been proposed in yeast to explain the role of PFDs in transcriptional elongation, although the identity of the complex is currently unknown (Millan-Zambrano et al., 2013). A PFDlike complex formed by PFD2 and PFD6 together with the PFD-like proteins URI1, UXT, PDRG1, and ASDURF has been identified in animals (Chaves-Perez et al., 2018). It is therefore reasonable to think that the putative Arabidopsis PFD-like complex may participate in PFDc-independent processes requiring PFD2 and/or PFD6. Defining the in vivo interactome of PFDs in different pfd mutant backgrounds would allow identifying putative alternative complexes and their partners, helping therefore to delineate PFDcindependent mechanisms for PFDs' action.

In summary, our results place PFDs as relevant players in the plant's response to environmental changes. Furthermore, the genetic analyses provide evidence that PFDs' action is not always mediated by the canonical PFDc, and clues about their possible mode of action in each case. The genetic resources generated in this work will help deciphering the mechanisms through which these versatile proteins act.

\section{Materials and methods \\ Plant material and growth conditions}

Arabidopsis thaliana accession Columbia-0 (Col-0) was used as wild type. Most mutants and transgenic lines have been described: pfd2 (Esteve-Bruna et al., 2020), pfd3 and pfd5 (Rodriguez-Milla and Salinas, 2009), pfd4 (Perea-Resa et al., 2017), pfd6-1 (Gu et al., 2008), RGApro::GFP-RGA (Silverstone et al., 2001), PFD4pro::PFD4-GFP (PereaResa et al., 2017), 35Spro::PFD6-YFP (Esteve-Bruna et al., 2020), and UBQ10pro::VENUS-TUA6 (Salanenka et al., 2018). The pfd1 mutant (GK-689A09) was obtained from the Nottingham Arabidopsis Stock Centre.

To grow seedlings in vitro, seeds were surface-sterilized, sown on plates with halfstrength MS (Duchefa) media, $\mathrm{pH} 5.7$, that includes $1 \%(\mathrm{w} / \mathrm{v})$ sucrose and $8 \mathrm{~g} \mathrm{~L}^{-1}$ agar (control media), and stratified at $4{ }^{\circ} \mathrm{C}$ for 3-4 days. Plates were exposed to continuous light $\left(50-60 \mu \mathrm{mol} \mathrm{m}{ }^{-2} \mathrm{~s}^{-1}\right)$ or LD photoperiod $\left(16 \mathrm{~h}\right.$ of $\left.90 \mu \mathrm{mol} \mathrm{m} \mathrm{m}^{-2} \mathrm{~s}^{-1}\right)$ at $22{ }^{\circ} \mathrm{C}$. For hypocotyl length measurements and TUA6-VENUS visualization, seedlings were grown without sucrose.

To obtain the $6 x$ pfd mutant, all double mutant combinations were first prepared by crossing individual $p f d$. Then, double mutants were used to obtain five triple mutants ( $p f d 1,2,3 ; p f d 1,3,5 ; p f d 2,3,5 ; p f d 2,4,6$, and $p f d 3,5,6)$. Triple mutants were used to obtain three quadruple mutants ( $p f d 1,2,3,5 ; p f d 1,3,5,6$, and $p f d 2,3,5,6)$. Crosses between triple and quadruple mutants, and between quadruples, were performed to obtain three quintuples (pfd1,2,3,5,6; pfd1,3,4,5,6, and $p f d 2,3,4,5,6)$ and the sextuple mutant. All mutant combinations were genotyped in F2 generations with primers listed in Table S1.

The UBQ10pro::VENUS-TUA6 pfd1 and UBQ10 pro::VENUS-TUA6 pfd2 lines were obtained by crossing and confirmed by genotyping in F2 or F3 generations with the primers listed in Table S1.

\section{Protein Structure Prediction}

The 3D structure of the Arabidopsis PFDc was modeled using the human PFDc (PDB code 6NR8) as template (Gestaut et al., 2019) using Modeller (release 9.23) (Webb and Sali, 2016), and visualized with PyMOL 2.4 software. 


\section{Tandem affinity purification}

The GS-PFD3 fusion under the control of the 35S promoter was generated and used to transform Arabidopsis PSB-D cell suspension cultures. The sequential affinity purification was performed as described (Van Leene et al., 2015). Proteolysis and peptide isolation, acquisition of mass spectra, and protein identification was carried out at the Unidad de Proteómica (CNB, Madrid, Spain).

\section{Gel filtration and Western Blot Analysis}

For gel filtration, protein extracts of 7-day-old PFD4pro::PFD4-GFP and 35Spro::PFD6YFP seedlings grown under continuous light were prepared in Extraction Buffer $(50 \mathrm{mM}$ Tris- $\mathrm{HCl} \mathrm{pH} \mathrm{7.5,} 150 \mathrm{mM} \mathrm{NaCl}, 10 \mathrm{mM} \mathrm{MgCl}_{2}, 10 \%$ glycerol, $0.5 \%$ Nonidet P-40, $2 \mathrm{mM}$ PMSF and 1x protease-inhibitor cocktail) and loaded onto a Superose ${ }^{\mathrm{TM}} 6$ Increase column (GE Healthcare). Twenty-four fractions of $0.5 \mathrm{~mL}$ were collected and precipitated as described (Esteve-Bruna et al., 2020). Proteins were separated in 12\% SDS-PAGE and transferred to a PVDF membrane by Western blotting. Membranes were stained with Ponceau S solution and then incubated with anti-GFP antibody (JL-8 Takara Bio Clontech, Lot \#A8034133).

To determine tubulin levels in pfd mutants, 7-day-old seedlings were grown under continuous light at $22^{\circ} \mathrm{C}$. To determine GFP-RGA, PFD4-GFP, and PFD6-YFP levels in the presence of salt or mannitol, 7-day-old seedlings grown in continuous light were exposed to $100 \mathrm{mM} \mathrm{NaCl}$ or $300 \mathrm{mM}$ mannitol in liquid media for 0,8 , or $24 \mathrm{~h}$. Ground frozen tissue from whole seedlings was homogenized in Extraction Buffer. Total proteins were separated in $12 \%$ SDS-PAGE, transferred to PVDF membranes by Western blotting and visualized with anti-GFP (JL-8 Takara Bio Clontech, Lot \#A8034133, 1:5000), anti- $\alpha$-tubulin (Invitrogen, Lot \#TD2479055, 1:1000), or anti-DET3 (1:10000, provided by Prof. Dr. Karin Schumacher). Quantification of protein bands in Western blots was performed using FIJI (https://fiji.sc/).

\section{Confocal microscopy}

To determine MT organization, seeds of UBQ10pro::VENUS-TUA6 in wild type, pfd1, and pfd2 backgrounds were germinated in MS media without sucrose and grown vertically in darkness for 3 days at $22^{\circ} \mathrm{C}$. MT were visualized in cells at the apical hook and immediately below by using a Nikon Eclipse Ti2 inverted microscope, equipped with a Yokogawa Spinning Disk Field Scanning Confocal System (https://www.microscope.healthcare.nikon.com/en_EU/products/confocal-

microscopes/csu-series/specifications). The objective used was the oil immersion CFI $60 \times$ H Plan Apocr $\lambda$ oil W.D. $0.13 \mathrm{~mm}$ N.A. 1.40. VENUS was excited by a $488 \mathrm{~nm}$ single mode optical fiber laser and the emission was collected at 525-550 $\mathrm{nm}$. Images were collected with a Photometrics Prime BSI CMOS camera (https://www.photometrics.com/products/prime-family/primebsi) with an exposure time of 100 to 200 ms with a $1 \times 1$ binning (2048 x 2048 pixels). The NIS-Element AR (Nikon, Japan, http://www.nis-elements.com/) was used as platform to control microscope, laser, camera, and post-acquisition analyses. Images were denoised by using the Denoise.ai algorithm (https://denoise.laboratory-imaging.com/process) and then analyzed using FIJI software.

\section{Phenotypic analyses}

The quantification of the sensitivity to oryzalin was carried out by measuring the length of the primary root of 7-day-old seedlings grown in LD on vertical plates supplemented with increasing concentrations of oryzalin $(0,75$, and $150 \mathrm{nM})$. Root length was measured using FIJI.

To determine rosette area, seeds were sown on 140-mm diameter Petri dishes at low density (40 seed per plate) and grown under continuous light. Plates were photographed 
bioRxiv preprint doi: https://doi.org/10.1101/2021.01.28.428673; this version posted January $29,2021$. The copyright holder for this preprint (which was not certified by peer review) is the author/funder, who has granted bioRxiv a license to display the preprint in perpetuity. It is made available under aCC-BY-NC-ND 4.0 International license.

with a digital camera 14 days after germination. The measurement was obtained using the FIJI plug-in Rosette tracker from at least 3 biological replicates (20 seedlings each). Representative images were taken with a Leica DMS1000 microscope. For hypocotyl length measurements, seeds were germinated in white light for $8 \mathrm{~h}$ and then grown in vertical plates in darkness for 7 days. Hypocotyl length was measured with FIJI.

For flowering-time measurements, seeds were sown on pots, stratified for 7 days at $4{ }^{\circ} \mathrm{C}$ and grown under SD ( $8 \mathrm{~h}$ light/16 h dark) or LD (16 h light/8 h dark) photoperiods at 22 ${ }^{\circ} \mathrm{C}$. Flowering time was recorded as the number of rosette and cauline leaves or days at bolting.

For the dormancy assay, seed lots to be compared were freshly harvested on the same day from individual plants grown in identical conditions. Seeds were sown immediately without stratification and incubated under continuous light. For the germination assay, freshly harvested seeds were sown and stratified for 3 days at $4{ }^{\circ} \mathrm{C}$. The percentage of seeds with an emerged radicle was determined at different time points.

\section{Stress Tolerance Assays}

$\mathrm{NaCl}, \mathrm{LiCl}$, and mannitol tolerance was analyzed by transferring 4-day-old seedlings grown on vertical MS plates under LD conditions to new MS plates supplemented with or without $100 \mathrm{mM} \mathrm{NaCl}, 12 \mathrm{mM} \mathrm{LiCl}$, or $300 \mathrm{mM}$ mannitol and incubated vertically for 4 days. Root length was measured using FIJI. Tolerance to freezing temperatures was determined as follows: 2-week-old plants grown on plates under LD photoperiod at 20 ${ }^{\circ} \mathrm{C}$ were transferred to $4{ }^{\circ} \mathrm{C}$ for 5 days and subsequently exposed to $-12{ }^{\circ} \mathrm{C}$ for 6 hours. Survival rates were determined after 1 week of recovering at $20^{\circ} \mathrm{C}$.

\section{RNA Extraction and Quantitative RT-PCR}

For expression analysis of flowering-related genes, seeds were sown on soil and grown in LD. Shoot apex and the second oldest leaf from 14-day-old plants were collected. For expression analysis of auxin-related genes, seedlings were grown for 5 days under continuous light at $22^{\circ} \mathrm{C}$ and then transferred to $29^{\circ} \mathrm{C}$ for $2 \mathrm{~h}$. For $P F D$ expression in the presence of salt or mannitol, 7-day-old seedlings grown in continuous light were exposed to $100 \mathrm{mM} \mathrm{NaCl}$ or $300 \mathrm{mM}$ mannitol in liquid media for 0,8 , or $24 \mathrm{~h}$. Total RNA was extracted using Machery-Nagel kit and treated with DNase I on column (MacheryNagel) following manufacturer's instructions. cDNA was synthesized with the PrimeScript ${ }^{\mathrm{TM}} 1 \mathrm{st}$ strand $\mathrm{cDNA}$ Synthesis Kit (Takara), and used as a template for qPCR assays employing the SYBR® Premix Ex Taq ${ }^{\mathrm{TM}}$ II (Takara) with primers listed in Table $\mathrm{S} 1$. The relative expression values were calculated using the At1g13320 (PDF2-1) gene as a reference, using the $\triangle \triangle C T$ method. All assays were performed with at least two biological replicates, each including three technical replicates.

For RNA-seq experiments, two type of samples were collected: (i) 7-day-old wild-type and $6 x$ pfd seedlings grown under continuous light at $22^{\circ} \mathrm{C}$, and (ii) wild-type and pfd 4 seedlings grown under LD conditions for 2 weeks at $22^{\circ} \mathrm{C}$. Total RNA was extracted with the RNeasy Plant Mini Kit (Qlagen), and the RNA concentration and integrity (RIN) were measured in a RNA nanochip (Bioanalyzer, Agilent Technologies 2100). The preparation of the libraries and the subsequent sequencing in an Illumina NextSeq ${ }^{\mathrm{TM}} 500$ platform was carried out by the Genomic Service of the University of Valencia.

\section{RNA-seq Analysis}

Read trimming was performed with cutadapt. Approximately, 20 million 75 bp paired-end reads per sample were generated and $>90 \%$ reads were aligned to the TAIR10 Col-0 reference genome using HISAT2 with default parameters. htseq-count was used for read counting and DESeq2 for identifiying DEGs as those that display absolute value of log2 fold chance $(\log \mathrm{FC})>1$ and $P$ adjusted value $<0.05$. Raw sequences (fastq files) and 
differential expression gene tables used in this paper have been deposited in the Gene Expression Omnibus (GEO) database (accession no. GSE138432). Raw data from previously published RNA-seq can be found in the same database with accession no. GSE124812.

Heatmaps from RNA-seq data were performed using the http://www1.heatmapper.ca/expression/ website. Volcano plots were constructed with the EnhancedVolcano R package. GO terms were obtained from AgriGO v2 and then were filtered with REVIGO for doing scatter plots. The Integrative Genomics Viewer was used to visualize reads from alignment files.

\section{Microarray-based Expression Analysis}

Data for PFD gene expression was gathered from the Bio-Analytic Resource Homepage Arabidopsis eFP Browser (http://bar.utoronto.ca/efp/cgi-bin/efpWeb.cgi). Heatmaps were generated using the Matrix2png tool (https://matrix2png.msl.ubc.ca/).

The coexpression analysis was done with the CORNET webtool (https://bioinformatics.psb.ugent.be/cornet/versions/cornet3.0/). Options used were: Pearson correlation method, correlation coefficient $>0.7$, retrieve top 170 genes, show pairwise correlations only. Microarray Compendium 1 TAIR10 (454 experiments with bias towards cell cycle, growth, and development) was used as source data.

\section{Statistical Analysis}

$P$ values in overlapping DEGs from two genotypes or conditions were calculated using hypergeometric tests. The rest of $P$ values were obtained from one-way ANOVA tests followed by multiple comparison tests when more than two genotypes were compared together. t-tests were performed instead when comparing only two genotypes.

\section{Acknowledgements}

We thank Dr. Jiri Friml (IST, Austria) for seeds of UBQ10::Venus-TUA6 and Dr. Karin Schumacher (University of Heidelberg, Germany) for the anti-DET3 antibody. Part of imaging analyses were carried out at NOLIMITS, an advanced imaging facility established by the University of Milan.

\section{Funding}

This work was supported by grants from the Spanish Ministry of Economy and Competitiveness and "Agencia Española de Investigación"/FEDER/European Union (BIO2013-43184-P to D.A. and M.A.B., and BIO2016-79133-P and PID2019-109925GB100 to D.A.). N.B-T. and A.S.-M. were recipient of a Ministerio de Economía y Competitividad (BES-2014-068868) and EU MSCA-IF (H2020-MSCA-IF-2016-746396) fellowships, respectively.

\section{References}

Amorim, A.F., Pinto, D., Kuras, L. and Fernandes, L. (2017) Absence of Gim proteins, but not GimC complex, alters stress-induced transcription. Biochim. Biophys. Acta - Gene Regul. Mech., 1860, 773-781.

Arranz, R., Martin-Benito, J. and Valpuesta, J.M. (2018) Structure and Function of the Cochaperone Prefoldin. Adv Exp Med Biol, 1106, 119-131.

Banks, C.A.S., Miah, S., Adams, M.K., Eubanks, C.G., Thornton, J.L., Florens, L. and Washburn, M.P. (2018) Differential HDAC1/2 network analysis reveals a role for prefoldin/CCT in HDAC1/2 complex assembly. Sci Rep, 8, 13712.

Bhattarai, K.K., Atamian, H.S., Kaloshian, I. and Eulgem, T. (2010) WRKY72-type transcription factors contribute to basal immunity in tomato and Arabidopsis as well as gene-for-gene resistance mediated by the tomato R gene Mi-1. Plant J., 63, 229-240. 
Blettinger, B.T., Gilbert, D.M. and Amberg, D.C. (2004) Actin up in the nucleus. Nat Rev Mol Cell Biol, 5, 410-415.

Bodt, S. De, Hollunder, J., Nelissen, H., Meulemeester, N. and Inzé, D. (2012) CORNET 2.0: Integrating plant coexpression, protein-protein interactions, regulatory interactions, gene associations and functional annotations. New Phytol., 195, 707-720.

Chaves-Perez, A., Thompson, S. and Djouder, N. (2018) Roles and Functions of the Unconventional Prefoldin URI. Adv Exp Med Biol, 1106, 95-108.

Choi, H. and Oh, E. (2016) PIF4 integrates multiple environmental and hormonal signals for plant growth regulation in Arabidopsis. Mol. Cells, 39, 587-593.

Delgehyr, N., Wieland, U., Rangone, H., et al. (2012) Drosophila Mgr, a prefoldin subunit cooperating with von Hippel Lindau to regulate tubulin stability. Proc. Natl. Acad. Sci. U. S. A., 109, 5729-5734.

Esteve-Bruna, D., Carrasco-López, C., Blanco-Touriñán, N., et al. (2020) Prefoldins contribute to maintaining the levels of the spliceosome LSM2-8 complex through Hsp90 in Arabidopsis. Nucleic Acids Res., 48, 6280-6293.

Fornara, F., Montaigu, A. de and Coupland, G. (2010) SnapShot: Control of flowering in arabidopsis. Cell, 141.

Franklin, K.A., Lee, S.H., Patel, D., et al. (2011) Phytochrome-Interacting Factor 4 (PIF4) regulates auxin biosynthesis at high temperature. Proc. Natl. Acad. Sci. U. S. A., 108, 20231-20235.

Fujioka, Y., Taira, T., Maeda, Y., Tanaka, S., Nishihara, H., Iguchi-Ariga, S.M.M., Nagashima, K. and Ariga, H. (2001) NM-1, a c-Myc-binding Protein, Is a Candidate for a Tumor Suppressor in Leukemia/Lymphoma and Tongue Cancer. J. Biol. Chem., 276, 45137-45144.

Geissler, S., Siegers, K. and Schiebel, E. (1998) A novel protein complex promoting formation of functional alpha- and gamma-tubulin. EMBO J, 17, 952-966.

Gendreau, E., Traas, J., Desnos, T., Grandjean, O., Caboche, M. and Hofte, H. (1997) Cellular basis of hypocotyl growth in Arabidopsis thaliana. Plant Physiol, 114, 295-305.

Gestaut, D., Roh, S.H., Ma, B., et al. (2019) The Chaperonin TRiC/CCT Associates with Prefoldin through a Conserved Electrostatic Interface Essential for Cellular Proteostasis. Cell, 177, 751-765 e15.

Gu, Y., Deng, Z., Paredez, A.R., DeBolt, S., Wang, Z.Y. and Somerville, C. (2008) Prefoldin 6 is required for normal microtubule dynamics and organization in Arabidopsis. Proc Natl Acad Sci U S A, 105, 18064-18069.

Huai, J., Zhang, X., Li, J., Ma, T., Zha, P., Jing, Y. and Lin, R. (2018) SEUSS and PIF4 Coordinately Regulate Light and Temperature Signaling Pathways to Control Plant Growth. Mol. Plant, 11, 928-942.

Irigoyen, M.L., Iniesto, E., Rodriguez, L., et al. (2014) Targeted degradation of abscisic acid receptors is mediated by the ubiquitin ligase substrate adaptor DDA1 in Arabidopsis. Plant Cell, 26, 712-728.

Kim, A.R. and Choi, K.W. (2019) TRiC/CCT chaperonins are essential for organ growth by interacting with insulin/TOR signaling in Drosophila. Oncogene, 38, 4739-4754.

Kleinboelting, N., Huep, G., Kloetgen, A., Viehoever, P. and Weisshaar, B. (2012) GABI-Kat SimpleSearch: New features of the Arabidopsis thaliana T-DNA mutant database. Nucleic Acids Res., 40, D1211-D1215.

Koini, M.A., Alvey, L., Allen, T., Tilley, C.A., Harberd, N.P., Whitelam, G.C. and Franklin, K.A. (2009) High temperature-mediated adaptations in plant architecture require the bHLH transcription factor PIF4. Curr Biol, 19, 408-413.

Kulkarni, S.R., Vaneechoutte, D., Velde, J. Van de and Vandepoele, K. (2018) TF2Network: predicting transcription factor regulators and gene regulatory networks in Arabidopsis using publicly available binding site information. Nucleic Acids Res., 46, e31. 
Kumar, S. V, Lucyshyn, D., Jaeger, K.E., Alos, E., Alvey, E., Harberd, N.P. and Wigge, P.A. (2012) Transcription factor PIF4 controls the thermosensory activation of flowering. Nature, 484, 242-245.

Lee, Y.S., Smith, R.S., Jordan, W., King, B.L., Won, J., Valpuesta, J.M., Naggert, J.K. and Nishina, P.M. (2011) Prefoldin 5 is required for normal sensory and neuronal development in a murine model. J. Biol. Chem., 286, 726-736.

Leene, J. Van, Eeckhout, D., Cannoot, B., et al. (2015) An improved toolbox to unravel the plant cellular machinery by tandem affinity purification of Arabidopsis protein complexes. Nat Protoc, 10, 169-187.

Leene, J. Van, Han, C., Gadeyne, A., et al. (2019) Capturing the phosphorylation and protein interaction landscape of the plant TOR kinase. Nat Plants.

Liang, J., Xia, L., Oyang, L., et al. (2020) The functions and mechanisms of prefoldin complex and prefoldin-subunits. Cell Biosci., 10, 87.

Lundin, V.F., Srayko, M., Hyman, A.A. and Leroux, M.R. (2008) Efficient chaperonemediated tubulin biogenesis is essential for cell division and cell migration in C. elegans. Dev. Biol., 313, 320-334.

March-Diaz, R. and Reyes, J.C. (2009) The beauty of being a variant: H2A.Z and the SWR1 complex in plants. Mol Plant, 2, 565-577.

Martin-Benito, J., Boskovic, J., Gomez-Puertas, P., Carrascosa, J.L., Simons, C.T., Lewis, S.A., Bartolini, F., Cowan, N.J. and Valpuesta, J.M. (2002) Structure of eukaryotic prefoldin and of its complexes with unfolded actin and the cytosolic chaperonin CCT. EMBO J, 21, 6377-6386.

Melki, R., Vainberg, I.E., Chow, R.L. and Cowan, N.J. (1993) Chaperonin-mediated folding of vertebrate actin-related protein and $\mathrm{y}$-tubulin. J. Cell Biol., 122, 13011310.

Millan-Zambrano, G., Rodriguez-Gil, A., Penate, X., Miguel-Jimenez, L. de, Morillo-Huesca, M., Krogan, N. and Chavez, S. (2013) The prefoldin complex regulates chromatin dynamics during transcription elongation. PLoS Genet, 9, e1003776.

Perea-Resa, C., Rodriguez-Milla, M.A., Iniesto, E., Rubio, V. and Salinas, J. (2017) Prefoldins Negatively Regulate Cold Acclimation in Arabidopsis Thaliana By Promoting Nuclear Proteasome-Mediated HY5 Degradation. Mol Plant, 10, 791804.

Quint, M., Delker, C., Franklin, K.A., Wigge, P.A., Halliday, K.J. and Zanten, M. van (2016) Molecular and genetic control of plant thermomorphogenesis. Nat Plants, 2, 15190 .

Ramegowda, V., Gill, U.S., Sivalingam, P.N., et al. (2017) GBF3 transcription factor imparts drought tolerance in Arabidopsis thaliana. Sci. Rep., 7, 9148.

Rodriguez-Milla, M.A. and Salinas, J. (2009) Prefoldins 3 and 5 play an essential role in Arabidopsis tolerance to salt stress. Mol Plant, 2, 526-534.

Salanenka, Y., Verstraeten, I., Löfke, C., Tabata, K., Naramoto, S., Glanc, M. and Friml, J. (2018) Gibberellin DELLA signaling targets the retromer complex to redirect protein trafficking to the plasma membrane. Proc. Natl. Acad. Sci. U. S. A., 115, 3716-3721.

Sangwan, V., Foulds, I., Singh, J. and Dhindsa, R.S. (2001) Cold-activation of Brassica napus BN115 promoter is mediated by structural changes in membranes and cytoskeleton, and requires Ca 2+ influx. Plant J., 27, 1-12.

Satou, A., Taira, T., Iguchi-Ariga, S.M. and Ariga, H. (2001) A novel transrepression pathway of c-Myc. Recruitment of a transcriptional corepressor complex to c-Myc by MM-1, a c-Myc-binding protein. J Biol Chem, 276, 46562-46567.

Siegers, K., Waldmann, T., Leroux, M.R., Grein, K., Shevchenko, A., Schiebel, E. and Hartl, F.U. (1999) Compartmentation of protein folding in vivo: Sequestration of non-native polypeptide by the chaperonin-GimC system. EMBO J., 18, 75-84.

Siegert, R., Leroux, M.R., Scheufler, C., Hartl, F.U. and Moarefi, I. (2000) Structure of the molecular chaperone prefoldin: unique interaction of multiple coiled coil 
tentacles with unfolded proteins. Cell, 103, 621-632.

Silverstone, A.L., Jung, H.S., Dill, A., Kawaide, H., Kamiya, Y. and Sun, T.P. (2001) Repressing a repressor: gibberellin-induced rapid reduction of the RGA protein in Arabidopsis. Plant Cell, 13, 1555-1566.

Skubacz, A., Daszkowska-Golec, A. and Szarejko, I. (2016) The role and regulation of ABI5 (ABA-insensitive 5) in plant development, abiotic stress responses and phytohormone crosstalk. Front. Plant Sci., 7, 1884.

Stortenbeker, N. and Bemer, M. (2019) The SAUR gene family: the plant's toolbox for adaptation of growth and development. J. Exp. Bot., 70, 17-27.

Sun, J., Qi, L., Li, Y., Chu, J. and Li, C. (2012) Pif4-mediated activation of yucca8 expression integrates temperature into the auxin pathway in regulating arabidopsis hypocotyl growth. PLoS Genet., 8, e1002594.

Vainberg, I.E., Lewis, S.A., Rommelaere, H., Ampe, C., Vandekerckhove, J., Klein, H.L. and Cowan, N.J. (1998) Prefoldin, a chaperone that delivers unfolded proteins to cytosolic chaperonin. Cell, 93, 863-873.

Wang, J., Gao, S., Peng, X., Wu, K. and Yang, S. (2019) Roles of the INO80 and SWR1 chromatin remodeling complexes in plants. Int. J. Mol. Sci., 20, 4591.

Webb, B. and Sali, A. (2016) Comparative protein structure modeling using MODELLER. Curr. Protoc. Bioinforma., 2016, 5.6.1-5.6.37.

Winter, D., Vinegar, B., Nahal, H., Ammar, R. and Wilson, G. V (2007) Electronic Fluorescent Pictograph" Browser for Exploring and Analyzing Large-Scale Biological Data Sets. PLoS One, 2, 718.

Zhou, F., Menke, F.L.H., Yoshioka, K., Moder, W., Shirano, Y. and Klessig, D.F. (2004) High humidity suppresses ssi4-mediated cell death and disease resistance upstream of MAP kinase activation, $\mathrm{H} 2 \mathrm{O} 2$ production and defense gene expression. Plant J., 39, 920-932.

\section{Figure legends}

Figure 1. The Arabidopsis PFDc. (a) Predicted structure of the Arabidopsis PFDc using the human PFDc as template for modelling. (b) Identification of the Arabidopsis PFDc in vivo. The table summarizes the average number of peptides and the Mascot score corresponding to each PFD subunit after TAP of GS-PFD3. (c) Gel filtration fractions were analyzed by Western blot and the fusion proteins revealed with anti-GFP antibodies.

Figure 2. Microtubule alterations in pfd mutants. (a) Representative confocal images of VENUS-TUA6 in wild-type, $p f d 1$, and pfd2 hypocotyl cells. Scale bar $=10 \mu \mathrm{m}$. (b) Effect of oryzalin on root elongation. The graph shows the average of two biological replicates. Error bars indicate standard error of mean. (c) Representative Western blot showing $\alpha-$ tubulin levels in the wild type and in pfd mutants. DET3 was used as loading control. The ratio of tubulin/DET3 of this representative blot is shown. (d) Levels of a-tubulin relative to DET3. Data are the average of five independent experiments. Three asterisks represent $P<0.001$ in Dunnet's multiple comparison test after ANOVA test. ${ }^{* * *}$ indicates $P<0.001$ in ANOVA tests when comparing all mutants with the WT.

Figure 3. Effect of pfd mutations in organ growth. (a) Representative images of 14-dayold rosettes of the indicated genotypes grown in $1 / 2$ MS plates under continuous light. Scale bar $=2 \mathrm{~mm}$. (b-d) Box plots showing the rosette area $(n \geq 52)$ in 14-day-old plants (b), the hypocotyl length after growing 7-day-old etiolated seedlings ( $n \geq 21)$ (c), and the root length $(n \geq 17)$ after growing 7 days in LD photoperiod (d). Horizontal lines inside boxes indicate the median. Whiskers indicate the highest and lowest values excluding outliers (points outside whiskers). Genotypes with different letters show significant differences at $P<0.05$ according to ANOVA with Tukey's HSD test. 
Figure 4. Flowering phenotype of pfd mutants. (a-b) Leaf number at bolting of plants grown in SD $(n \geq 9)(a)$ or in LD $(n=12)(b)$. Open and filled bars represent rosette and cauline leaves, respectively. Error bars indicate standard deviation of each kind of leaves. ${ }^{*}$ and ${ }^{* * *}$ indicate $P<0.05$ and 0.001 , respectively, in Dunnet's multiple comparison tests after ANOVA tests when the total number of leaves is considered. (c) Major pathways controlling flowering time in Arabidopsis. (d) Summary of qRT-PCR results. Circles indicate the genes analyzed in the apex shoot and /or the $2^{\text {nd }}$ oldest leaf of 14-d-old wild-type and 6x pfd plants grown in LD. (e) Expression of misregulated genes in $6 x$ pfd plants compared to the wild type. Data are mean from 3 biological replicates. Error bars represent standard error from means. One, two, and three asterisks represent $P<0.05,0.01$, and 0.001 in t-tests.

Figure 5. Cold acclimation in pfd mutants. (a) Freezing tolerance assay of coldacclimated plants. Two-day-old wild-type, pfd1, pfd2, pfd3, pfd4, pfd5, pfd6, and 6x pfd plants grown at $20^{\circ} \mathrm{C}$ were transferred to $4{ }^{\circ} \mathrm{C}$ for 5 days and subsequently exposed to $-12^{\circ} \mathrm{C}$ for $6 \mathrm{~h}$. Survival rates were determined after 1 week of recovering at $20^{\circ} \mathrm{C}$. Error bars indicate standard error of mean from five biological replicates. One, two, and three asterisks indicate $P<0.05,0.01$, and 0.001 , respectively, in Dunnet's multiple comparison tests after ANOVA tests. (b) Representative plates after recovery. (c) Threedimensional reconstruction of the Arabidopsis PFDc using pfd6-1 instead of PFD6. The R-to-Q amino acid substitution in pfd6-1 is highlighted in red.

Figure 6. Response salt and osmotic stress in pfd mutants. (a-c) Root length in the presence of $100 \mathrm{mM} \mathrm{NaCl}$ (a), $12 \mathrm{mM} \mathrm{LiCl}$ (b), or (c) $300 \mathrm{mM}$ mannitol. Bars represent mean from eight $(a, c)$ or three $(b)$ biological replicates. Error bars indicate standard error of mean. One and three asteriks indicate $P<0.01$ and 0.001 , respectively, in Dunnet's multiple comparison tests after ANOVA.

Figure 7. Transcriptomic analysis of the $6 x$ pfd mutant. (a) Volcano plots highlighting the DEGs in $6 x$ pfd and pfd4 mutants (only genes with $\geq 1$ RPKM in three replicates of mutants and/or wild type are shown). (b) Venn diagram comparing DEGs in pfd4 and $6 x$ pfd mutants. (c) Cytoscape image of the coexpression network of DEGs in the $6 x$ pfd mutant. Red and blue indicates down-and upregulated genes, respectively. (d) Plots showing the expression level of the indicated genes extracted from the RNA-seq analysis. Each dot represents a replicate.

Figure 8. Novel functions for PFDs. (a) Heatmap showing the behavior of auxin related genes misregulated in $6 x$ pfd seedlings. Asterisks mark SAUR genes included in cluster 3. (b) Expression of auxin-related genes in response to 2 hours at $29^{\circ} \mathrm{C}$. Data are mean of 3 biological replicates \pm standard error of mean. Different letters show significant differences at $P<0.05$ according to ANOVA with Newman-Keuls tests. (c, d) Germination rates of non-stratified (c) and stratified (d) wild-type and $6 x$ pfd seeds. Error bars represent standard error of mean from two biological replicates (each including 4060 seeds). One, two, and three asterisks represent $P<0.05,0.01$, and 0.001 in Bonferroni tests after ANOVA tests, respectively. ns = no significant differences.

Figure 9. Constitutive stress signature in $6 x$ pfd seedlings. (a) Venn diagram comparing DEGs in the 6x pfd mutant and in the wild type exposed to either salt or cold stress. (b) Heatmap showing the behavior of common DEGs in the three conditions. (c) GO terms enriched in the 163 common DEGs. Only some GO terms are represented. All GO terms are shown in Table S4. Bubble size is proporcional to the $P$ significance of the GO enrichment.

Figure S1. Structure of the human and Arabidopsis PFDc. The structure of the different Arabidopsis and human PFDs is shown in (a). PFDN1 to PFDN6 and PFD1 to PFD6 
refer to the human and Arabidopsis PFDs, respectively. The 3D structure of Arabidopsis and human PFDc is shown in (b). The 3D structure of the Arabidopsis PFDc was modelled by using the human PFDc as template.

Figure S2. The novel pfd1 mutant is a null allele. (a) Structure of the PFD1 gene indicating the position of the T-DNA insertion (triangle). Boxes and lines indicate exons and introns, respectively. White boxes correspond to the $5^{\prime}$ and $3^{\prime}$ untranslated regions. Arrows indicate the position of oligonucleotides used for the semi-quantitative RT-PCR analysis of PFD1 expression. (b) Semi-quantitative RT-PCR analysis of PFD1 expression in wild-type (WT) and pfd1 plants. A negative control (water) was also included. PCR products were amplified with primers indicated in Table S1.

Figure S3. Expression of PFD genes in 6x pfd. (a) IGV plots showing the RNA-seq average reads density (three replicates) in PFD genes in the wild type (black) and 6x pfd (purple). Purple triangles and a red arrow indicate the position of insertions and the pfd61 mutation, respectively. (b) The $6 x$ pfd seedlings used for RNA-seq were homozygous for the pfd5 insertion. (c) Electrophoregram showing the position of the T-DNA insertion in $p f d 5$.

Figure S4. pfd mutant plants grown on soil. Representative images of 21-day-old plants of the indicated genotypes grown under LD conditions are shown. Scale bar $=2 \mathrm{~cm}$.

Figure S5. Heatmap showing the expression levels of the six PFD genes at different stages of Arabidopsis development.

Figure S6. The $6 x$ pfd mutant flowers early. (a) Flowering time of wild-type and $6 x$ pfd plants $(n \geq 7)$ under LD, measured as the number of days for bolting. Three asterisks represent $P<0.001$ in a t-test. (b,c) Expression analysis of flowering-time regulator genes in shoot apex (b) or the $2^{\text {nd }}$ oldest rosette leaf (c). Data are mean from 3 biological replicates. Error bars represent standard error from means. One asterisk represents $P<$ 0.05 in t-test.

Figure 7. PFDs' transcripts or proteins do not respond to salt stress. (a) Levels of PFD1 to PFD6 transcripts in 7-day-old seedlings grown in continuous light and exposed to 100 $\mathrm{mM} \mathrm{NaCl}$ for 0,8 or 24 hours. Values represent mean from 3 technical replicates. Error bars represent the standard deviation of these replicates. NCED and HKT1 were used as control for the $\mathrm{NaCl}$ treatment. A second biological replicate showed equivalent results. (b) Levels of GFP-RGA, PFD4-GFP, and PFD6-YFP fusion proteins in 7-day-old seedlings grown in the same conditions than in (a).

Figure S8. PFDs' transcripts or proteins do not respond to osmotic stress. (a) Levels of PFD1 to PFD6 transcripts in 7-day-old seedlings grown in continuous light and exposed to $300 \mathrm{mM}$ mannitol for 0,8 or 24 hours. Bars represent mean from 3 technical replicates. $R D 26$ was used as control of the temperature treatment. A second biological sample showed equivalent results. (b) Levels of PFD4-GFP and PFD6-YFP proteins in 7-dayold seedlings grown in the same conditions than in (a).

Figure S9. RNA-seq analysis of $6 x$ pfd seedlings. (a) Heatmap showing the behavior of the 81 common DEGs of $6 x$ pfd and pfd 4 mutants grown in control conditions. (b) A magnification of the 3 clusters shown in Figure 3c. Pink edges mean correlation 0.7-0.8, light blue edges man correlation 0.8-0.9, and dark blue edges mean correlation 0.9-1. (c) GO terms enriched in DEGs in the 6x pfd mutant. Only some GO terms are represented. All GO terms are listed in Supplementary File 1. Bubble size is proporcional to the $P$ significance of $\mathrm{GO}$ enrichment. 
Figure S10. PFDs' transcript levels do not change in responseto warm temperatures. Levels of PFD1 to PFD6 transcripts in 5-day-old seedlings grown in continuous light at $22^{\circ} \mathrm{C}$ and exposed to $29^{\circ} \mathrm{C}$ for 2 hours. Values represent mean from 3 technical replicates. Error bars represent the standard deviation of these replicates. A second biological sample showed equivalent results. HSP70 was used as control for the temperature treatment.

Figure S11. The PFDc contributes to seed germination. Germination rates of stratified seeds. Error bars represent standard error of mean from seven biological replicates (each one including at least 29 seeds). Three asterisks represent $P<0.001$ in Bonferroni tests after ANOVA tests; when comparing with the wild type, all pfd mutants show this significant difference at $24 \mathrm{~h}$, while at $32 \mathrm{~h}$ the pfd 1 shows no significant differences. $n s$, no significant differences.

Figure S12. Constitutive stress signature in $6 x$ pfd seedlings. The Venn diagram shows the overlap between DEGs. Heatmaps show the behavior of DEGs.

Figure S13. PFD4 is involved in the regulation of the gene expression in response to cold and salt stresses. Venn diagrams showing the overlaps between DEGs in the wild type and pfd4 seedlings in response to high salt (a) or low temperature (b). 
(a)

(b)

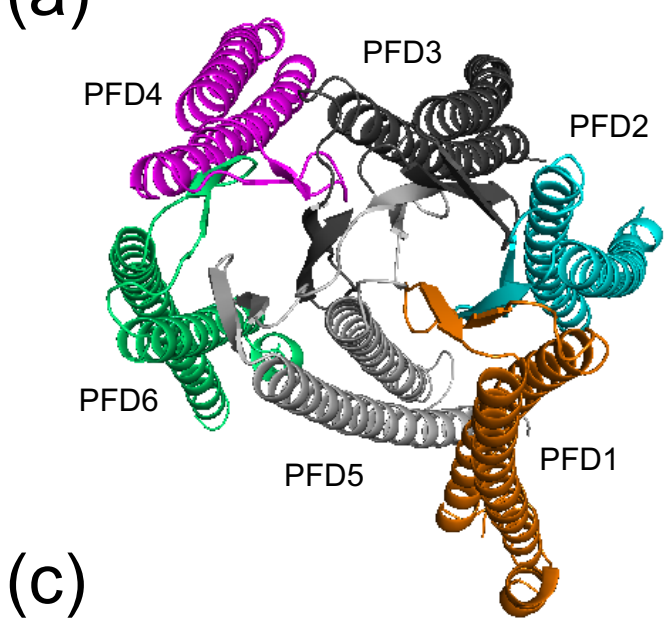

\begin{tabular}{ccc}
\hline Interactor & No. of peptides & Score \\
\hline PFD3 & 473 & 164.5 \\
\hline PFD2 & 436 & 137.6 \\
\hline PFD6 & 234 & 119.0 \\
\hline PFD5 & 150 & 139.5 \\
\hline PFD4 & 110 & 122.9 \\
\hline PFD1 & 71 & 123.1 \\
\hline
\end{tabular}

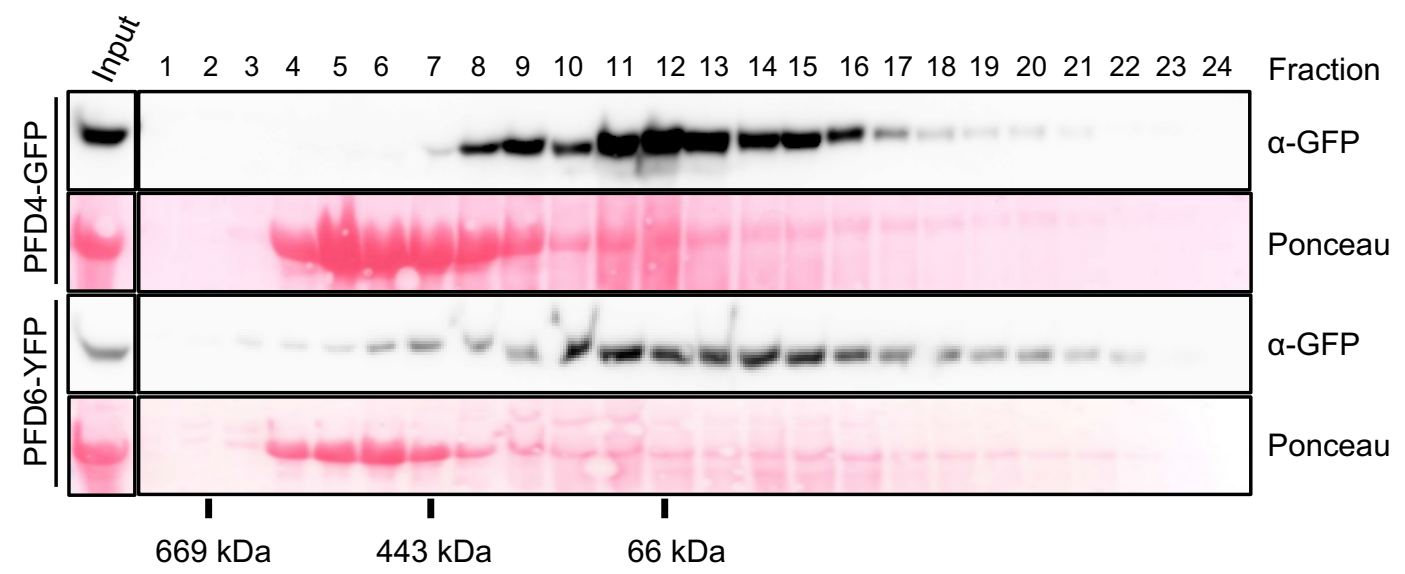

Figure 1 
(a)

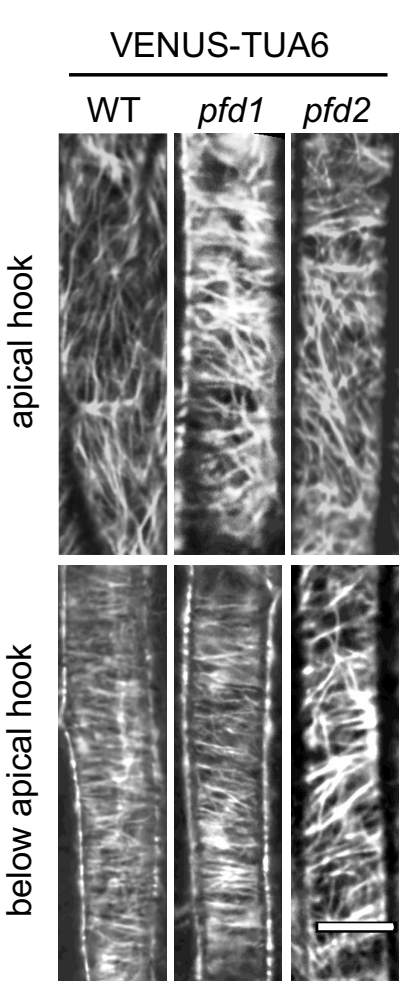

(c)

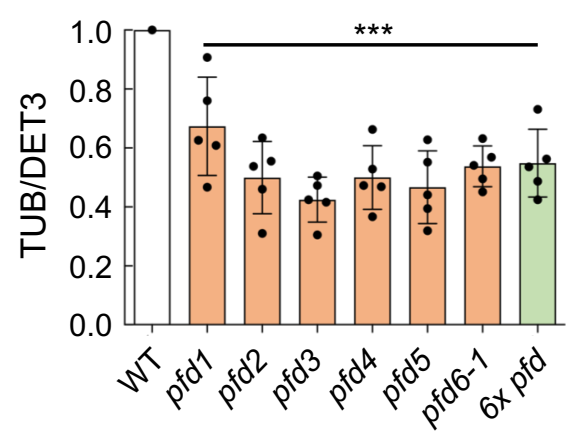

(b)

- WT $\triangle p f d 2 \quad \diamond p f d 4 \quad \diamond p f d 6-1$

$\neg p f d 1 \quad \neg-\nabla f d 3 \quad-0-p f d 5 \quad-0-6 x p f d$

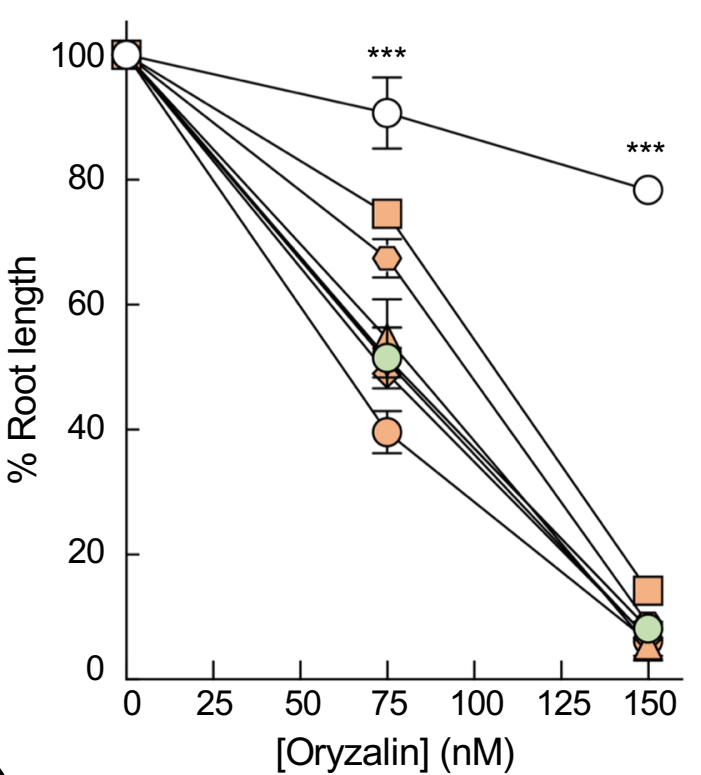

(d)

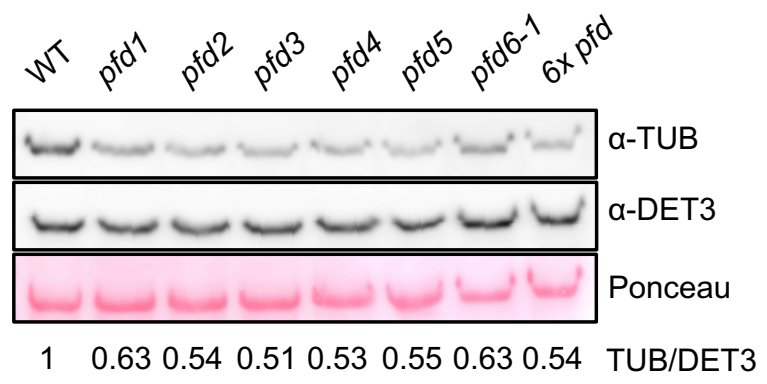


(a)

(b)
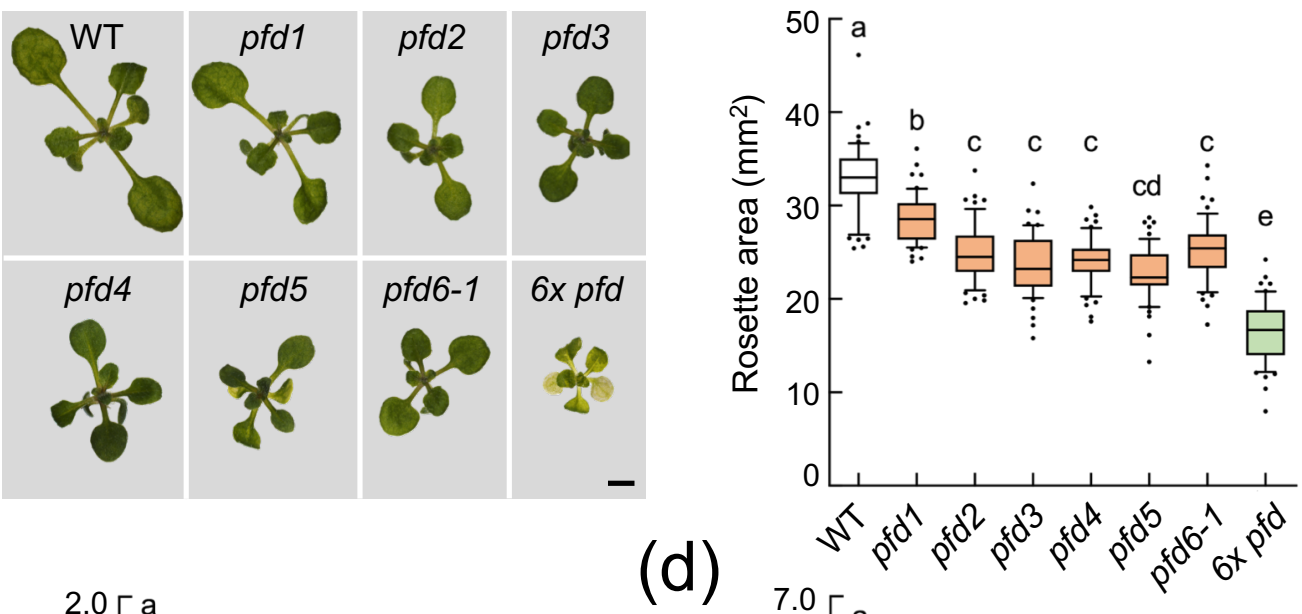

(c)
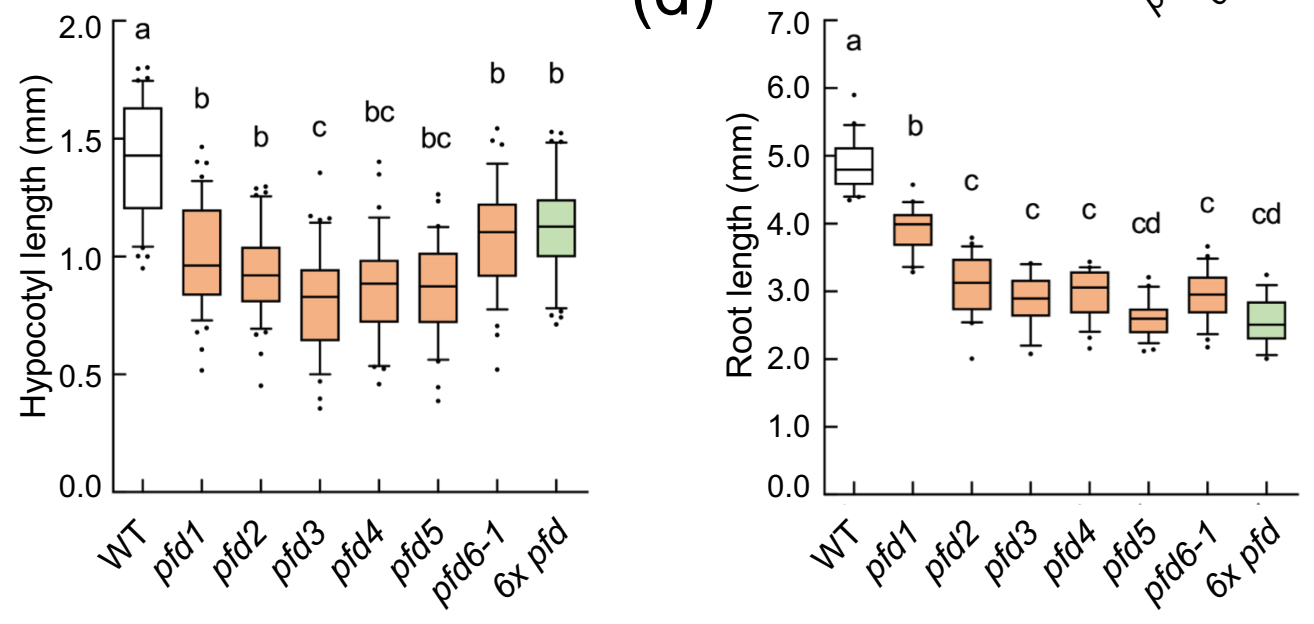

Figure 3 
(a)

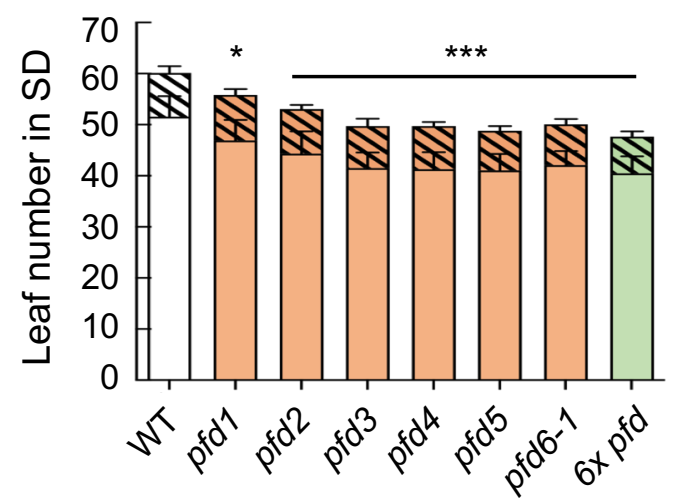

(c)

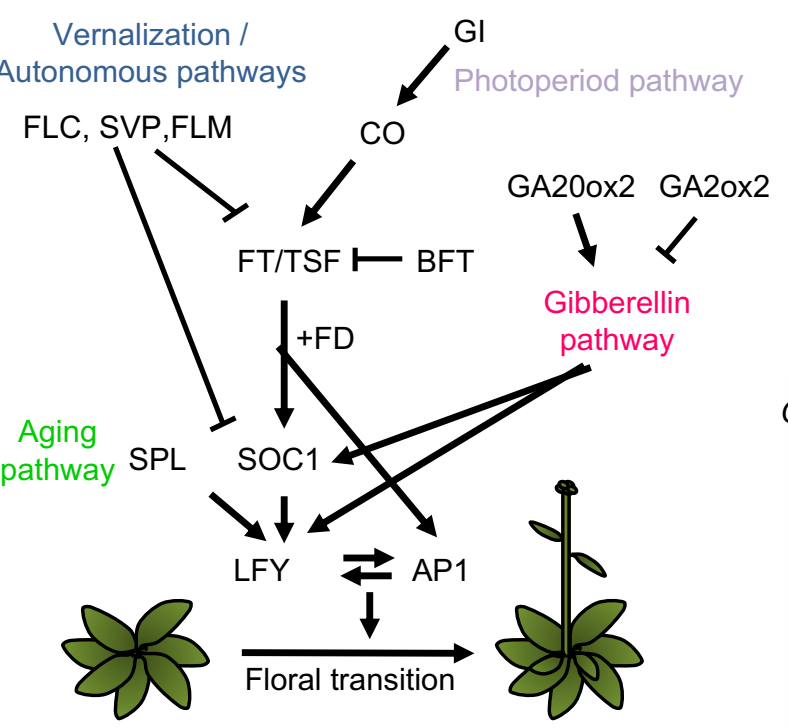

(e)

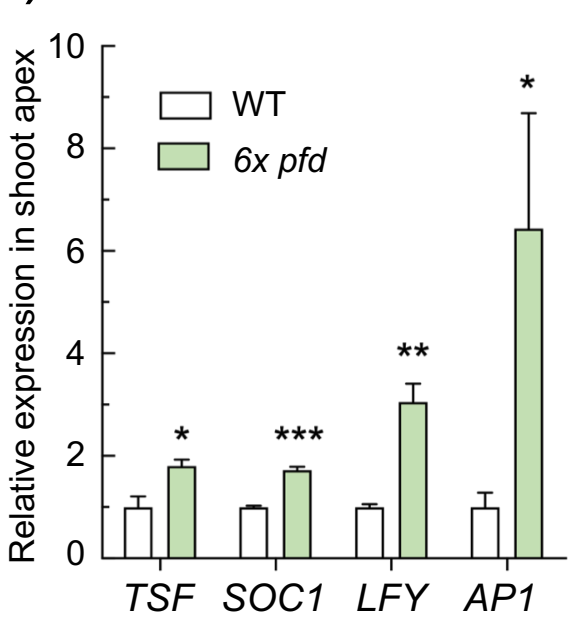

(b)

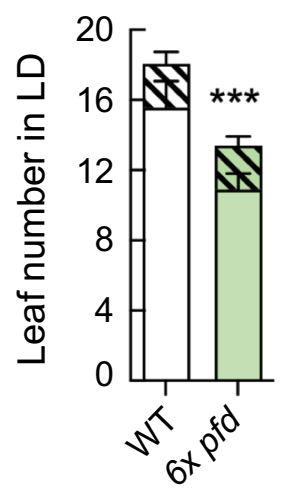

(d)

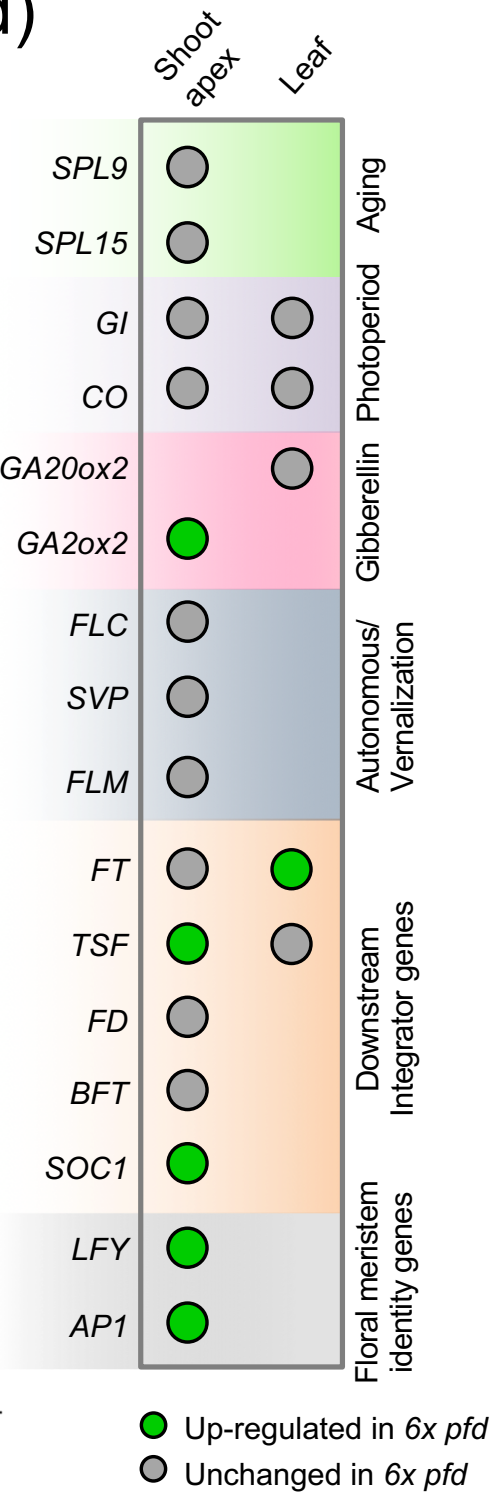

Figure 4 
(a)

(c)
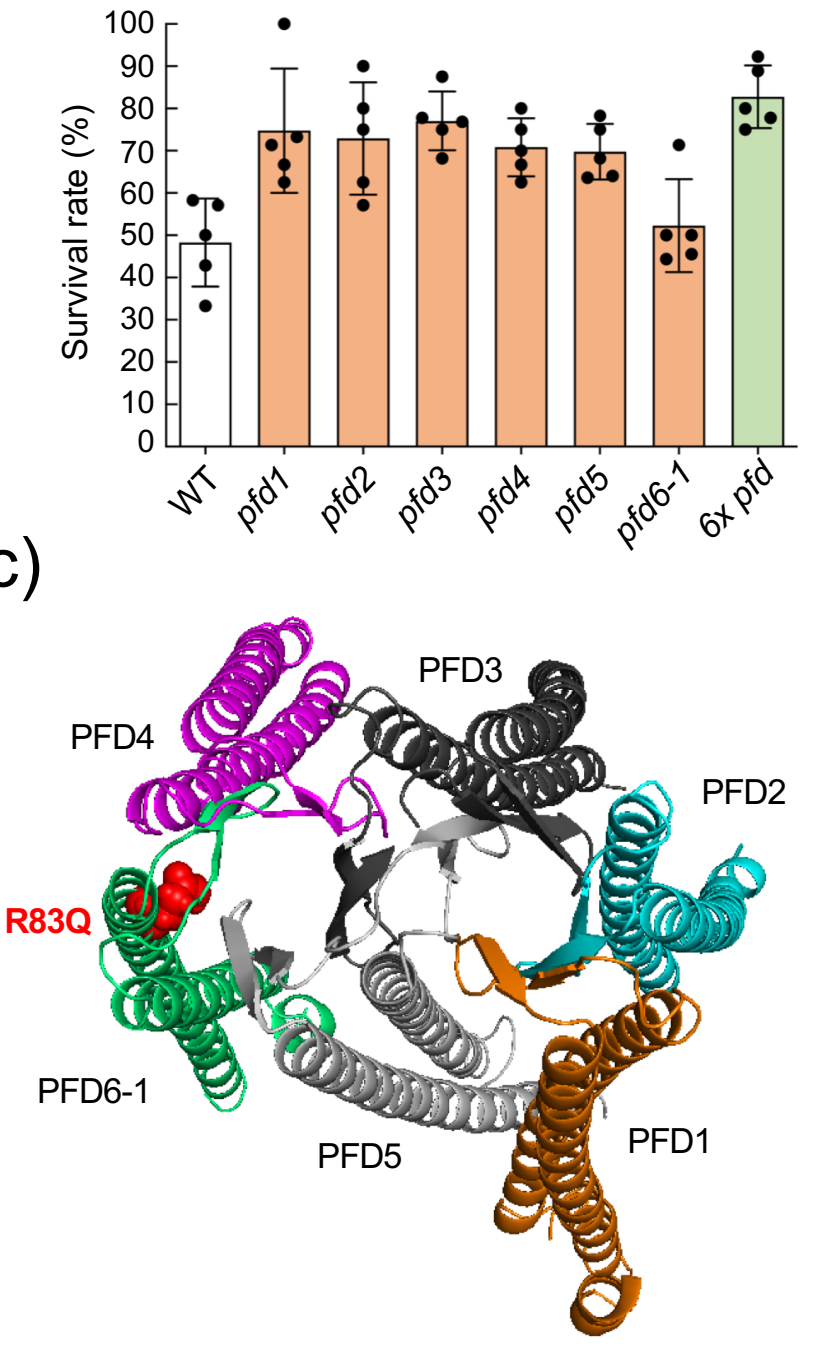

(b)
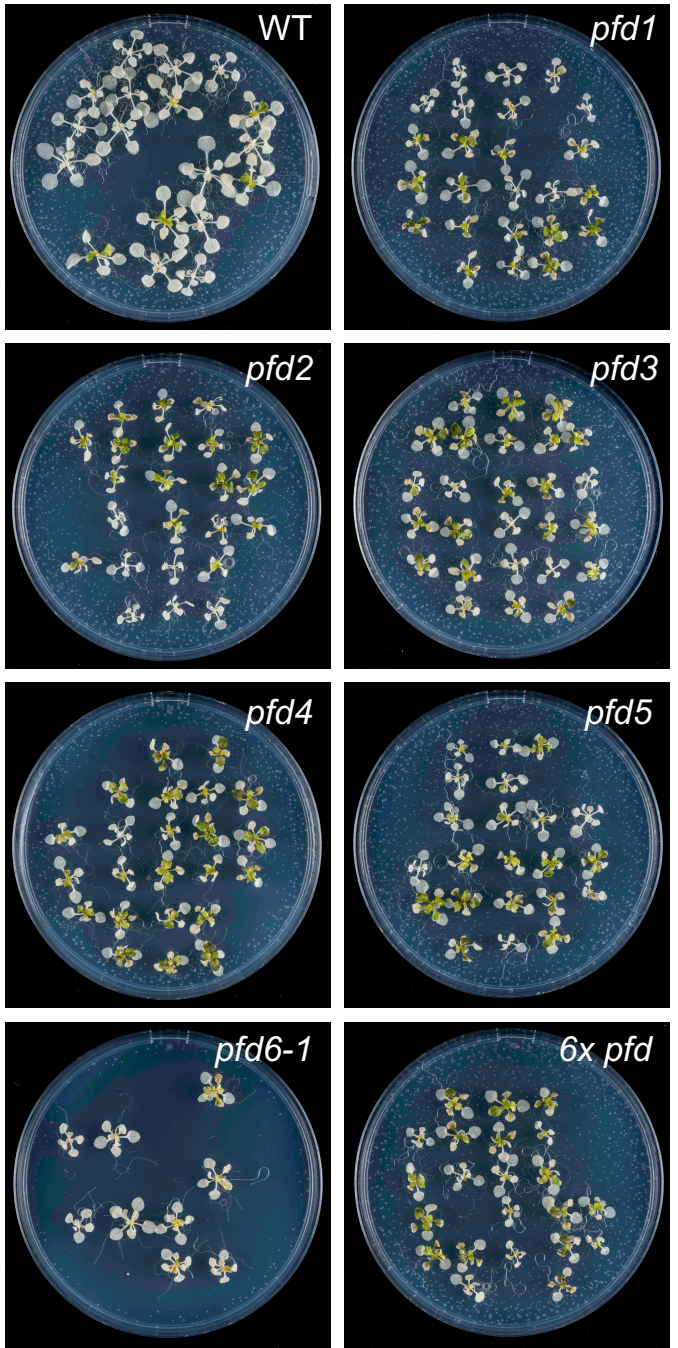

Figure 5 
(a)

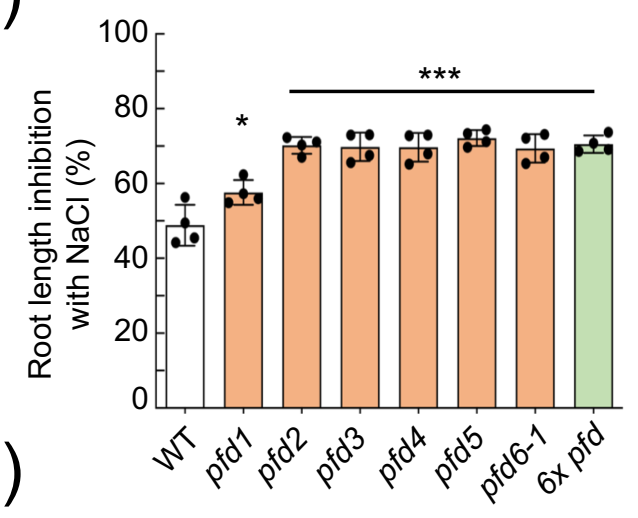

(b)

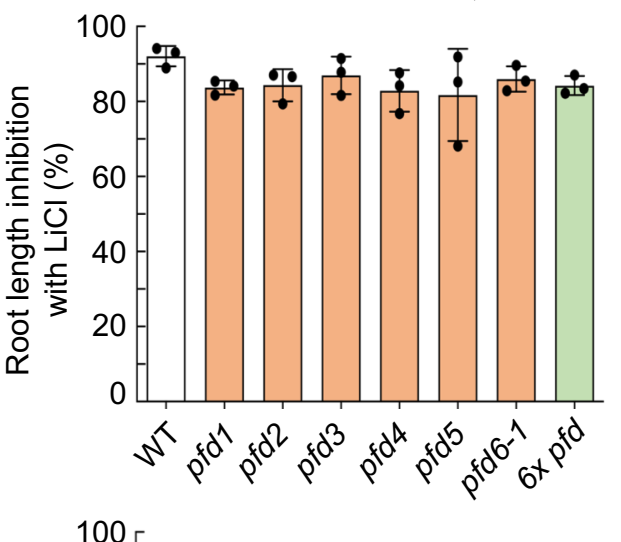

(c)

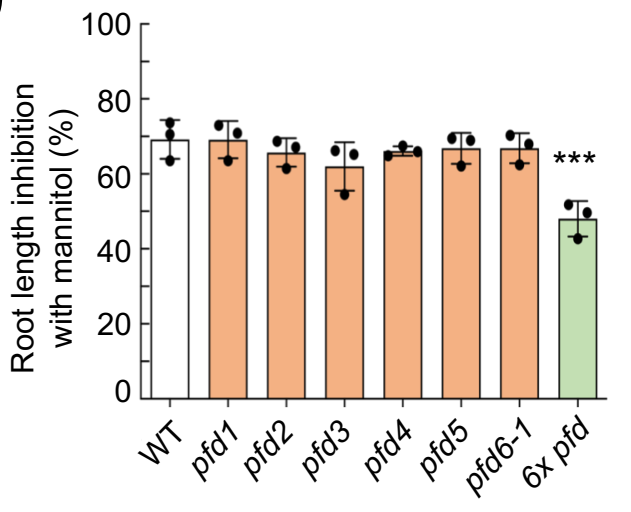

Figure 6 
(a)
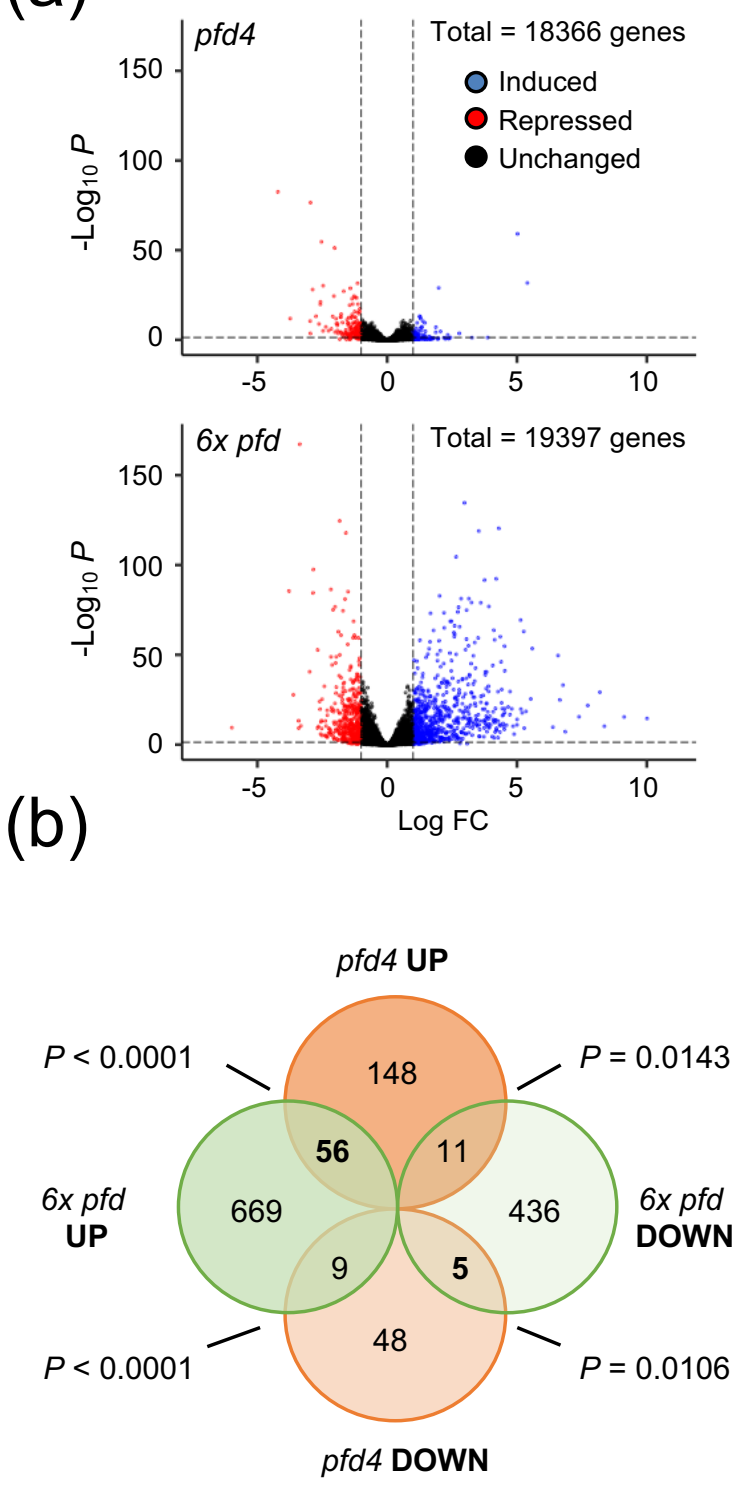

(c)

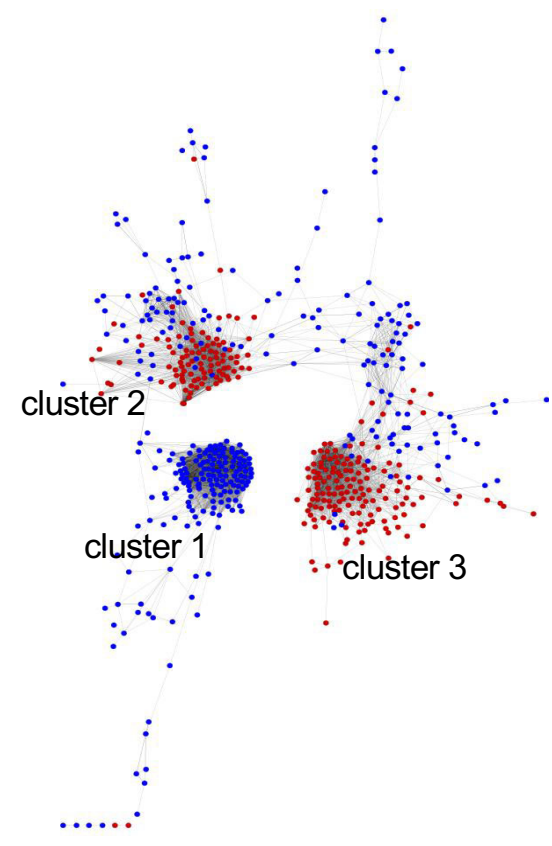

(d)

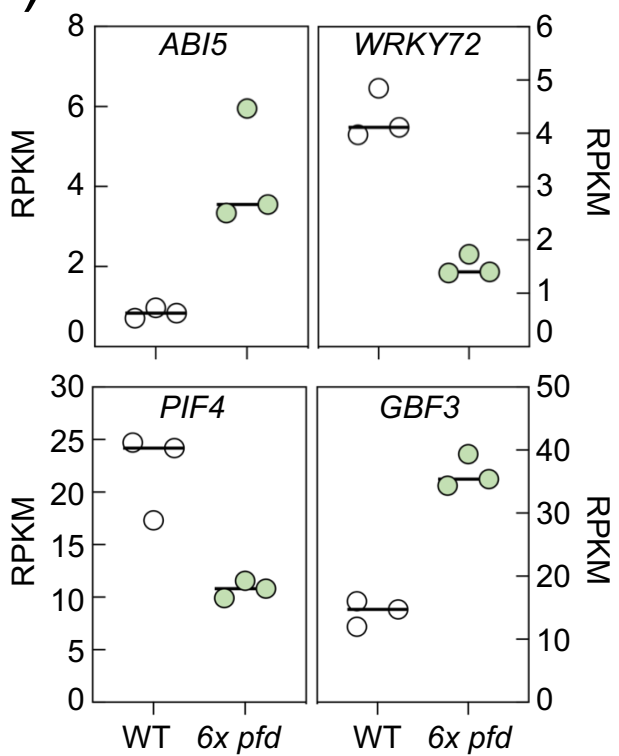


(a)

(b)
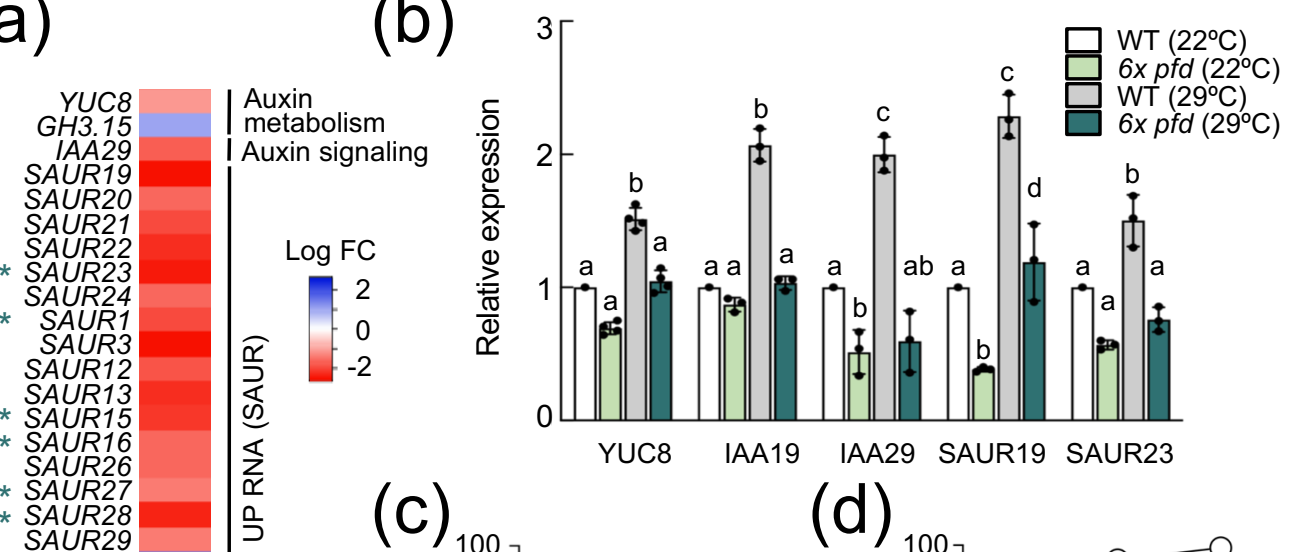

(c)

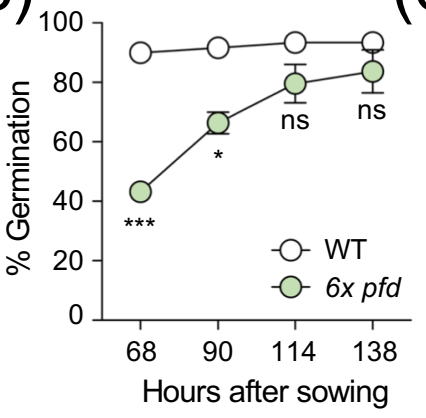

(d)

SAUR35

SAUR50

SAUR56

SAUR61

SAUR62

SAUR63

SAUR64

SAUR66

SAUR67

SAUR74

SAUR76

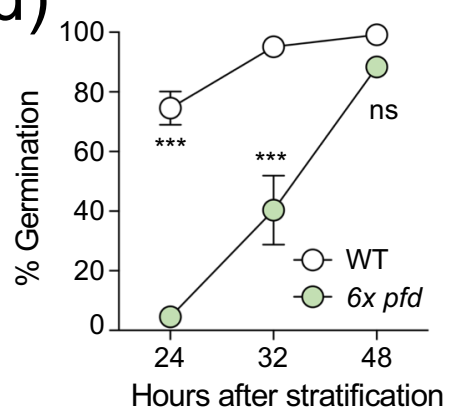

Figure 8 
(a)

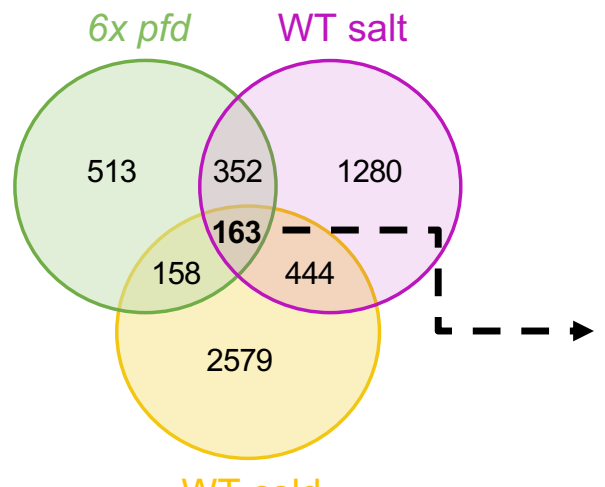

(c)

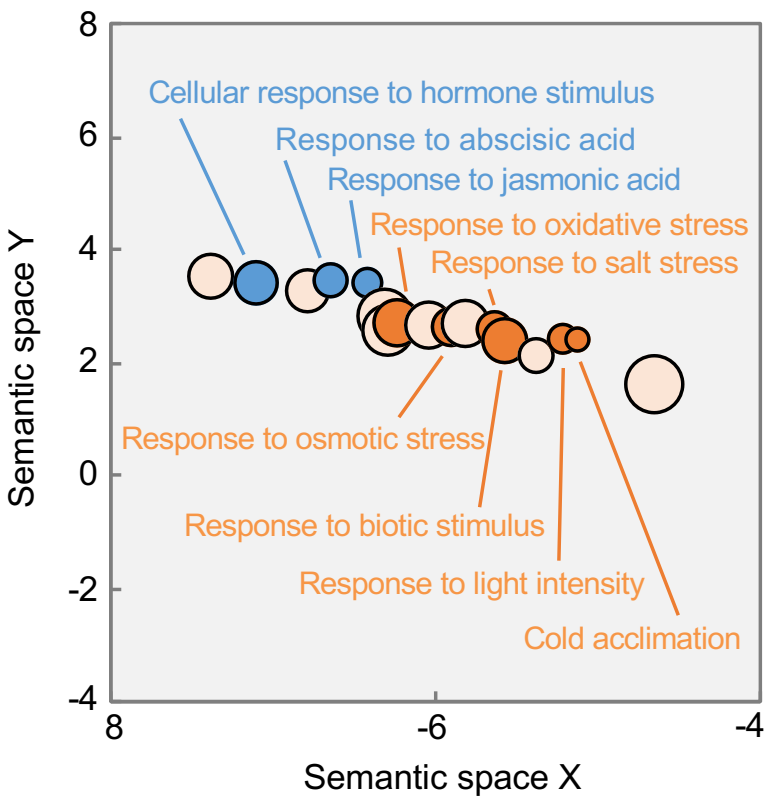

(b)

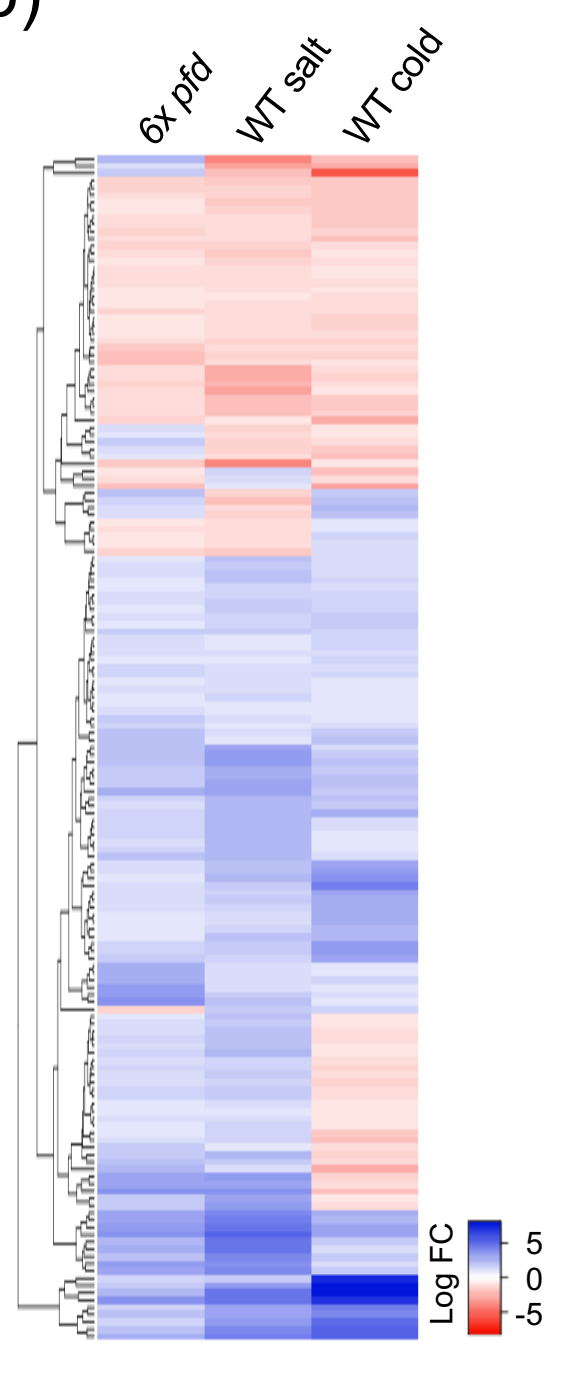

Figure 9 
(a)
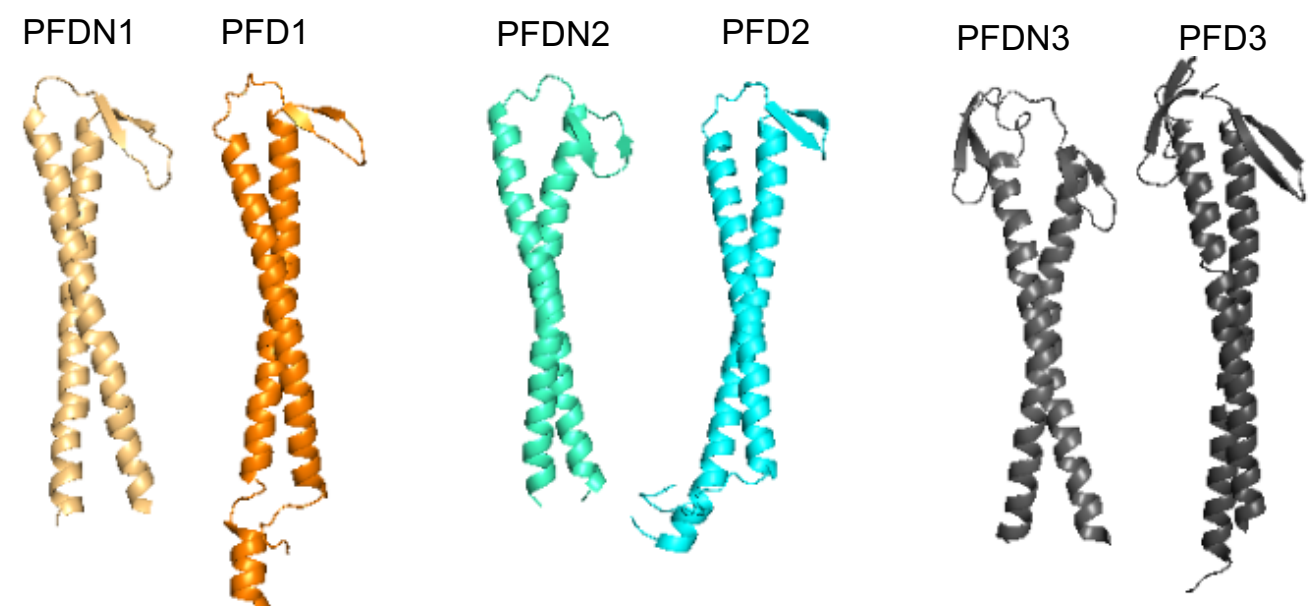

PFDN4 PFD4
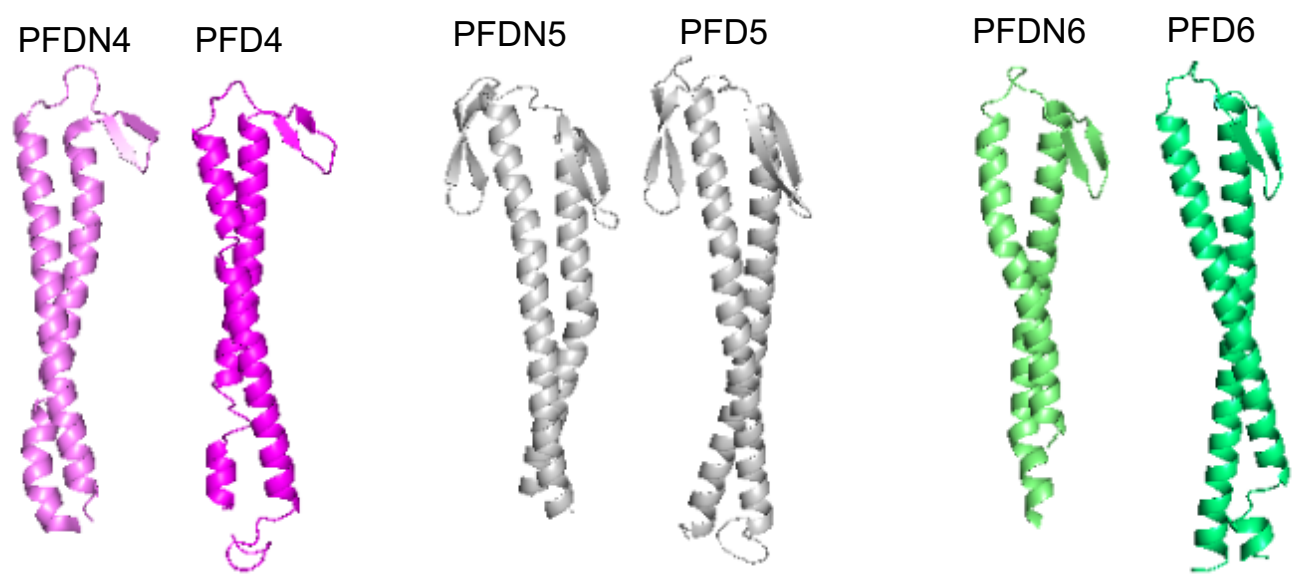

(b)

Human PFDc

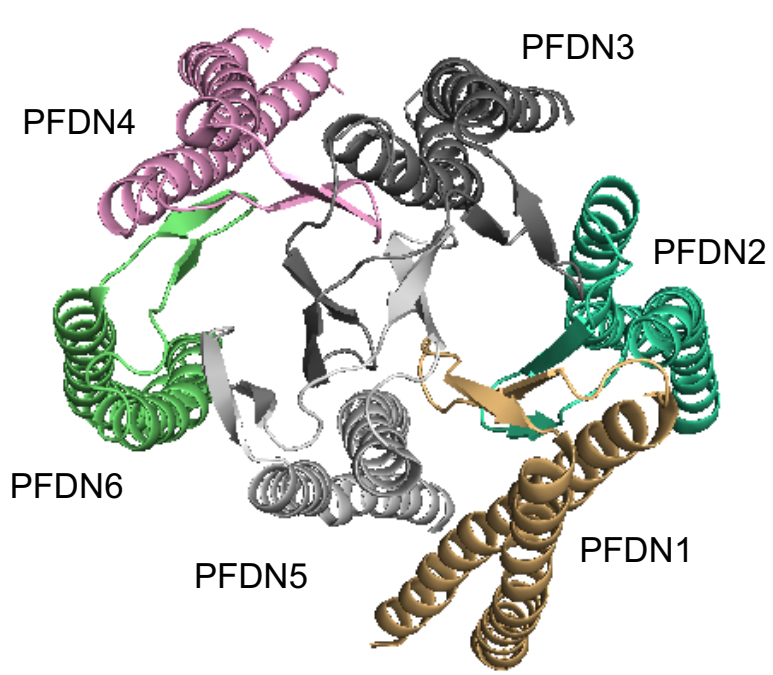

Arabidopsis PFDc

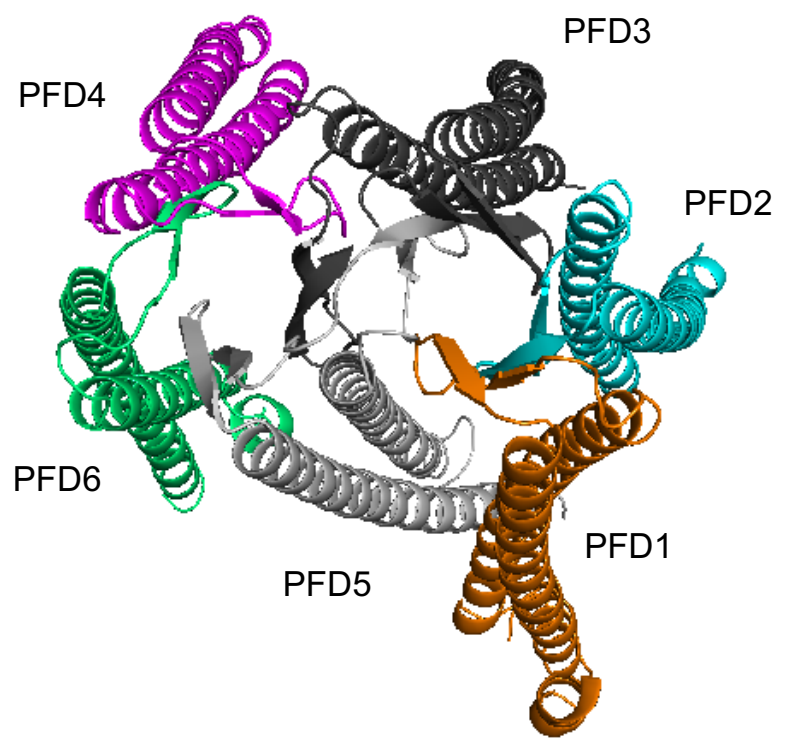

Figure S1 
(a)

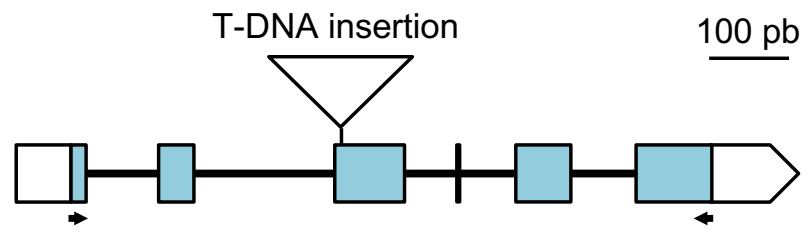

(b)

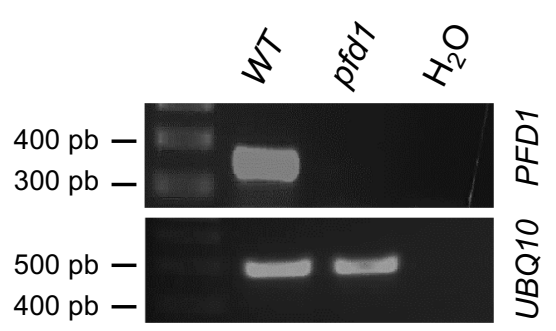

Figure S2 
(a)
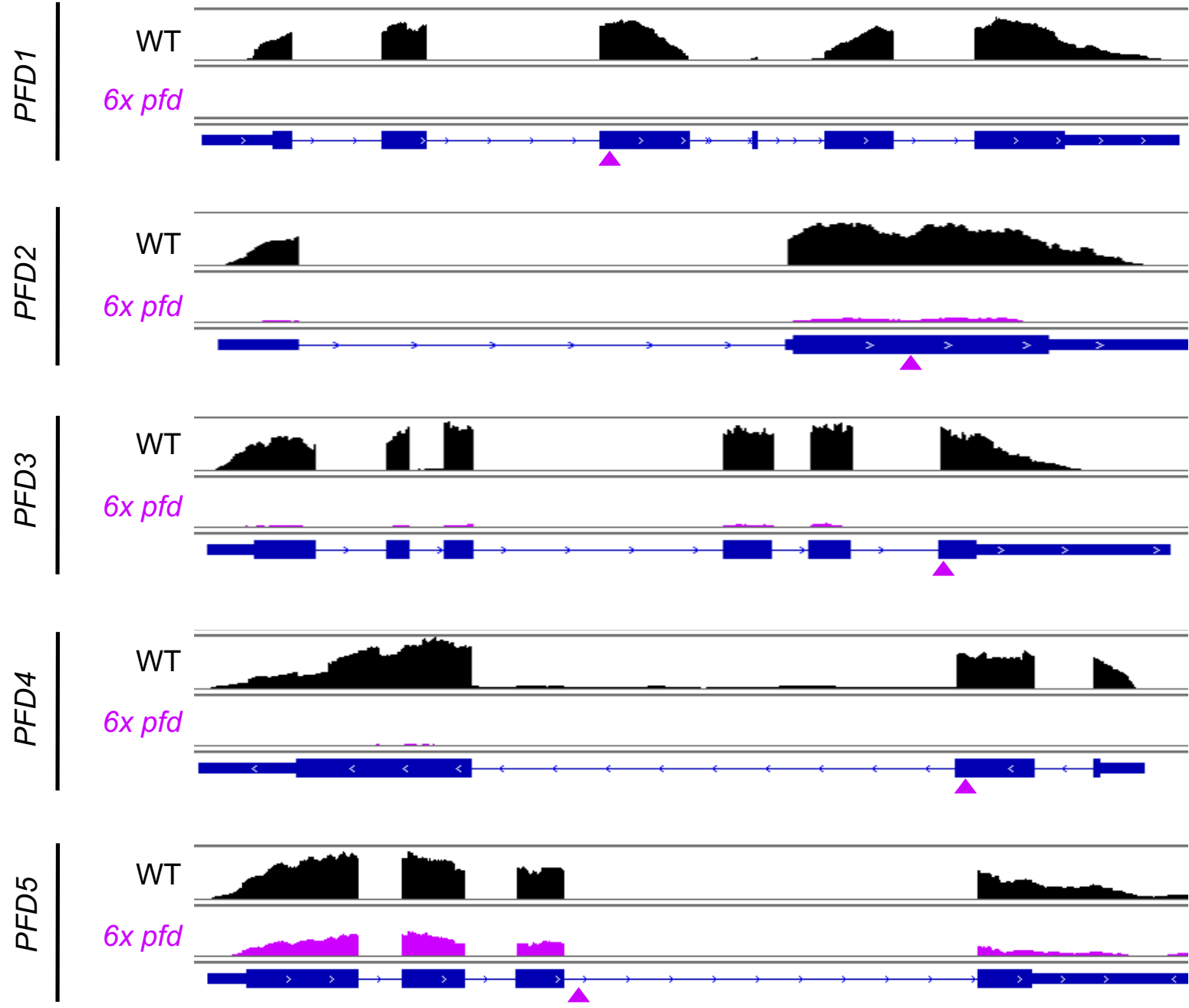

(b)
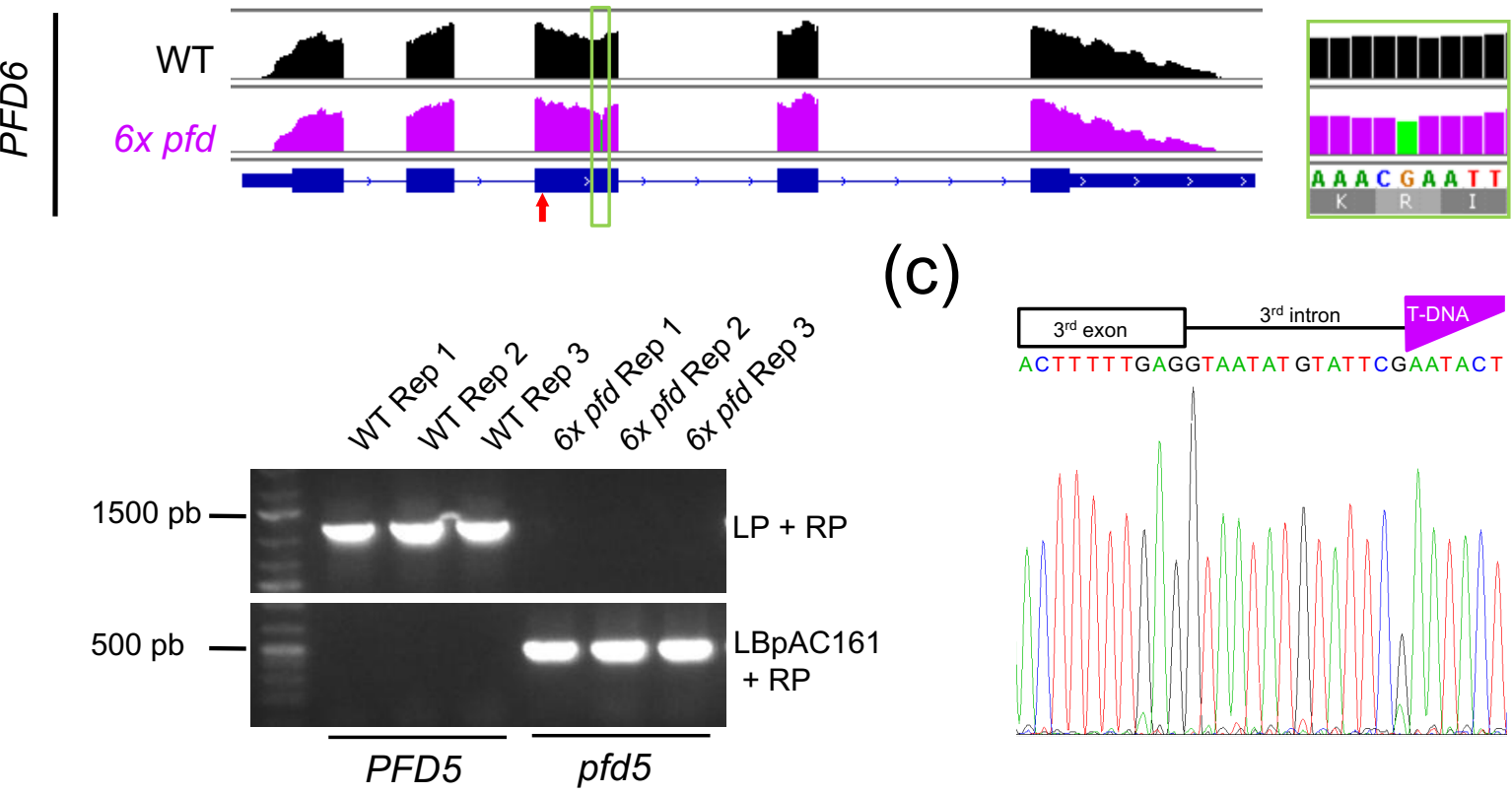

(c)

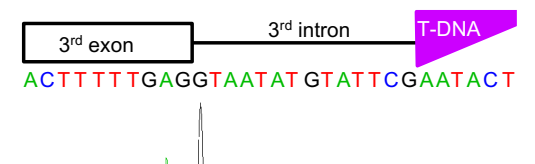

Figure S3 


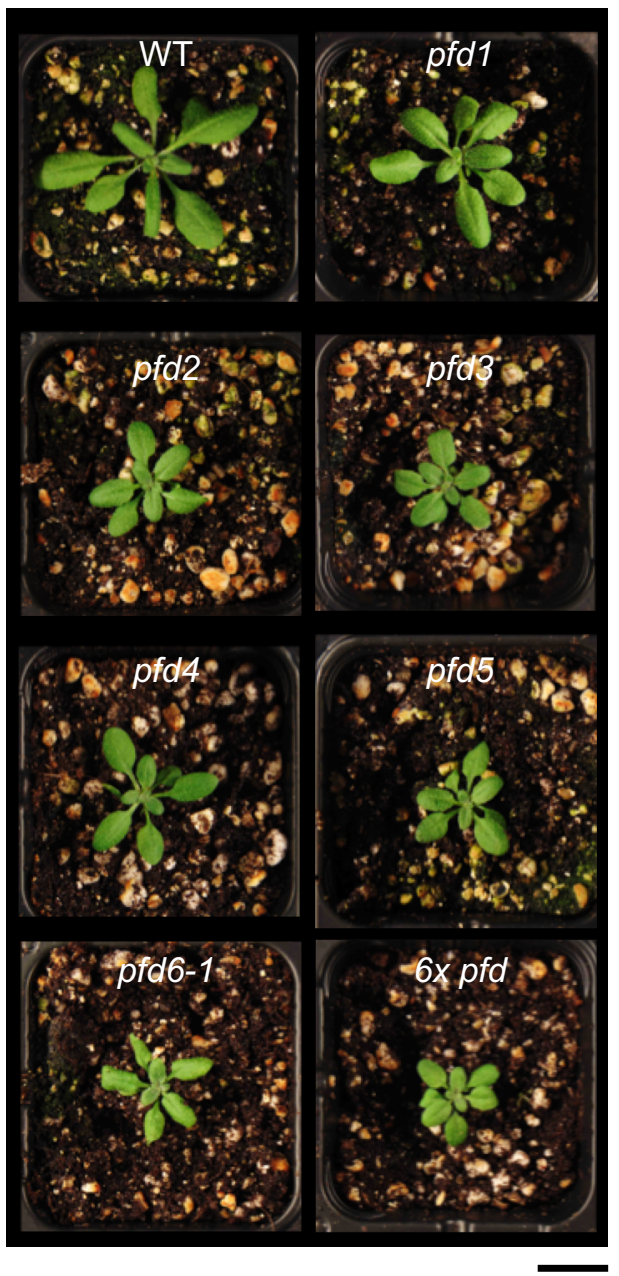

Figure S4 


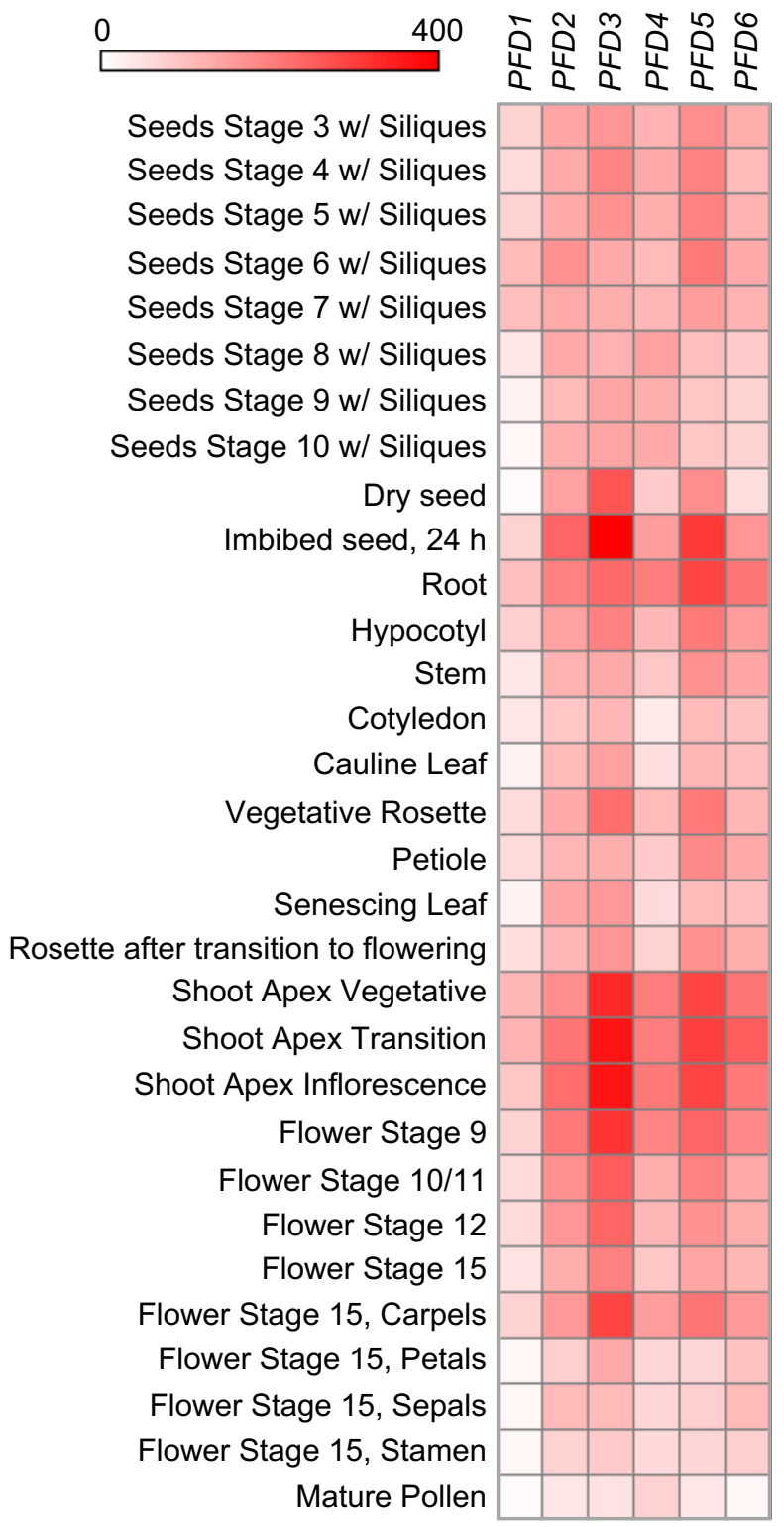

Figure S5 
(a)

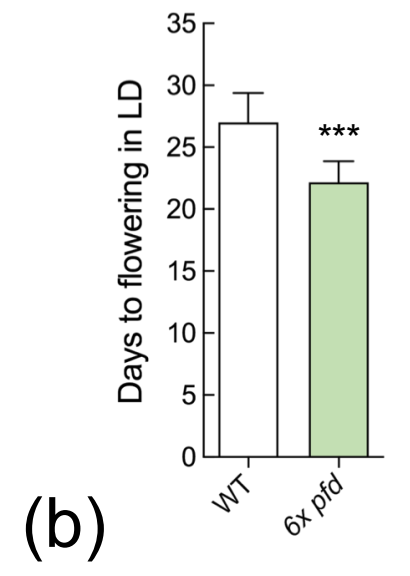

(c)
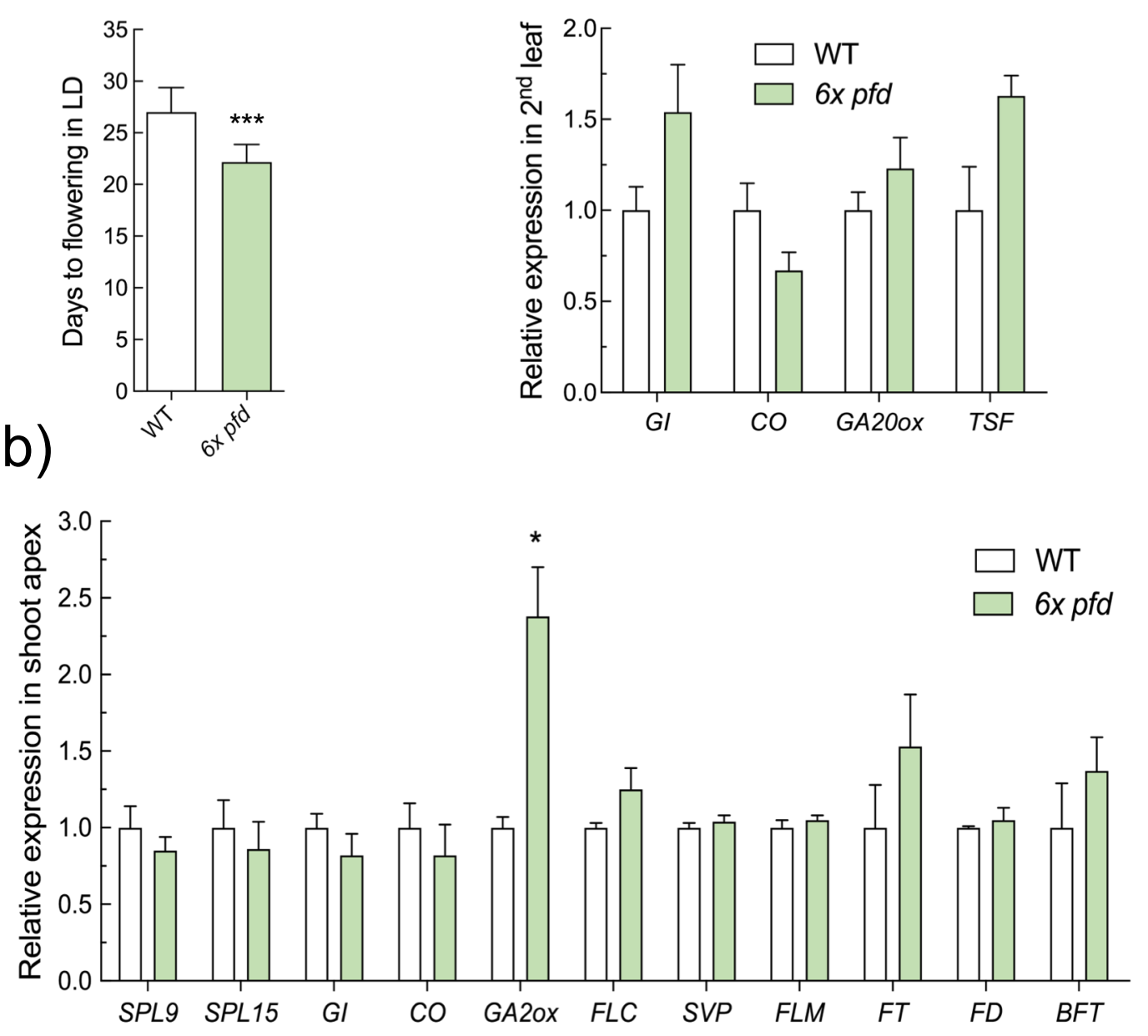
(a)

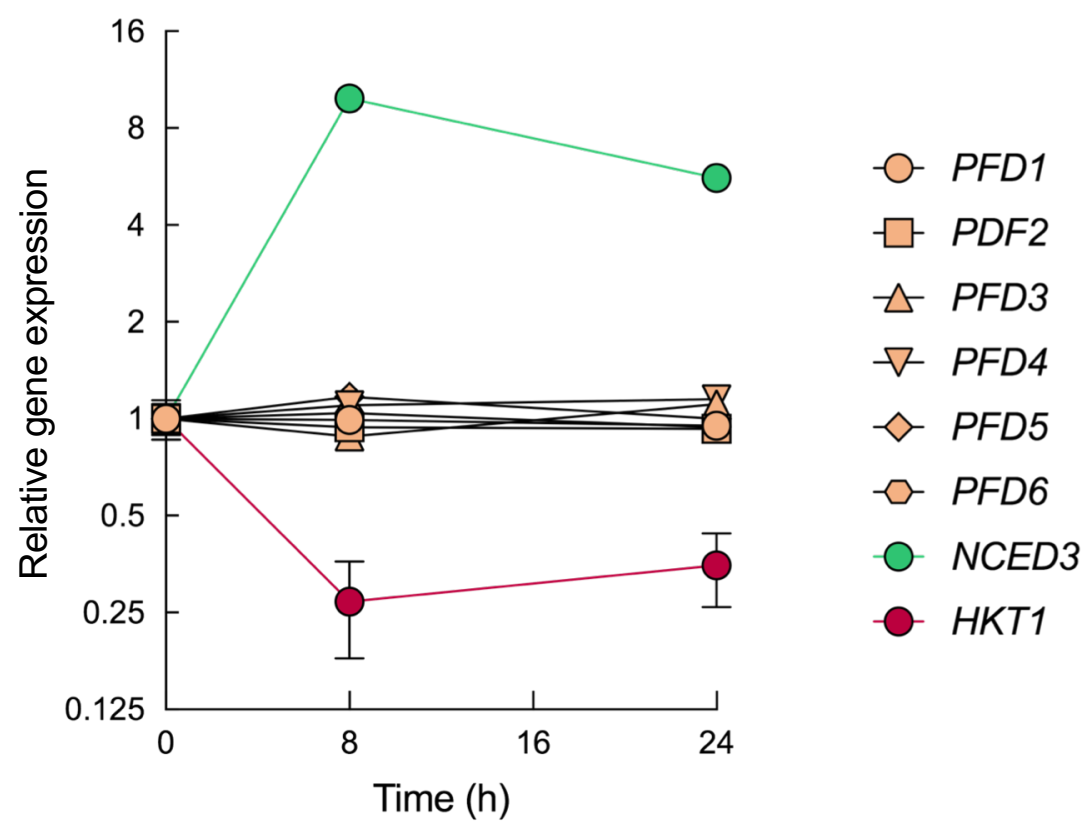

(b)

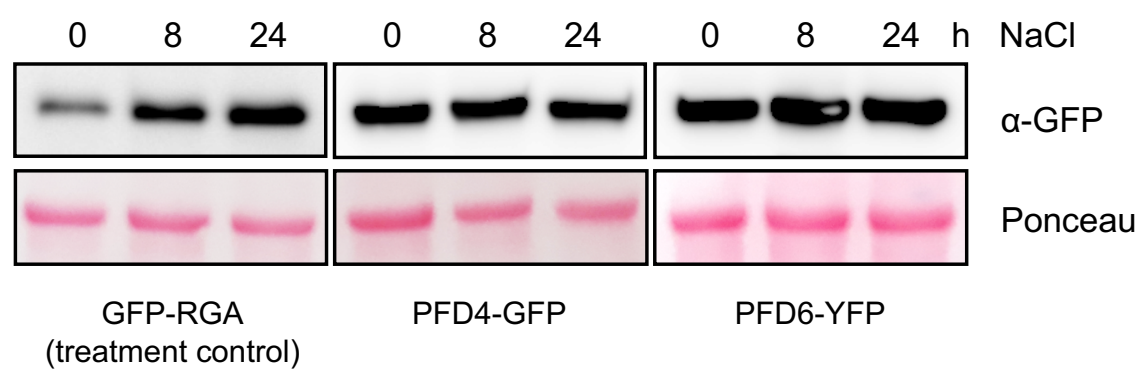

Figure S7 
(a)

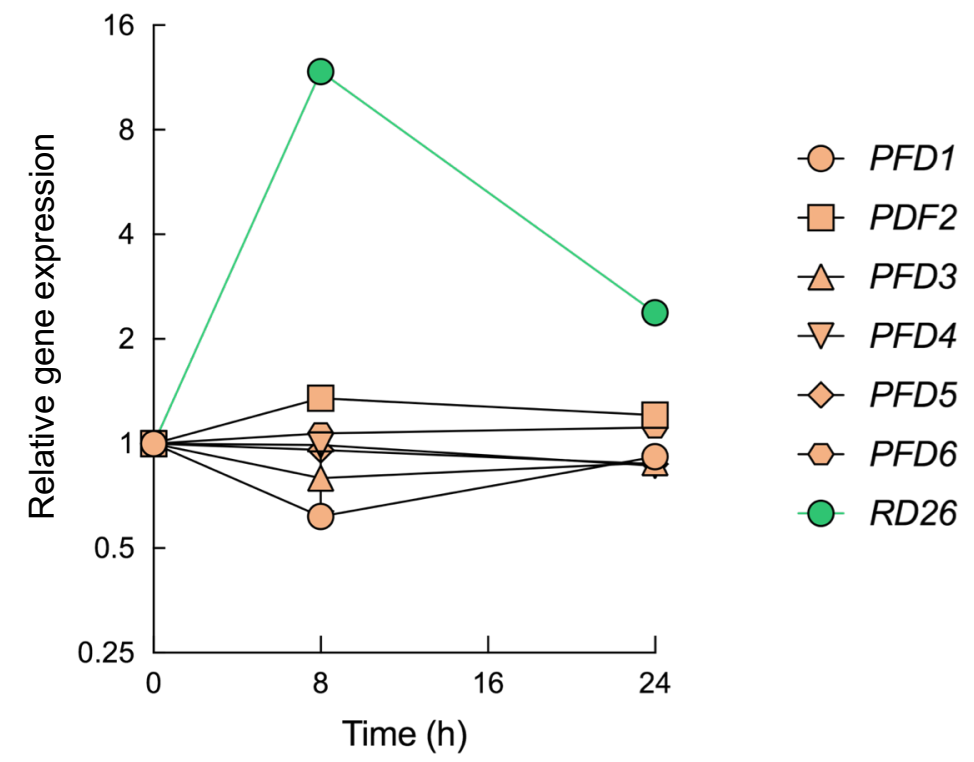

(b)

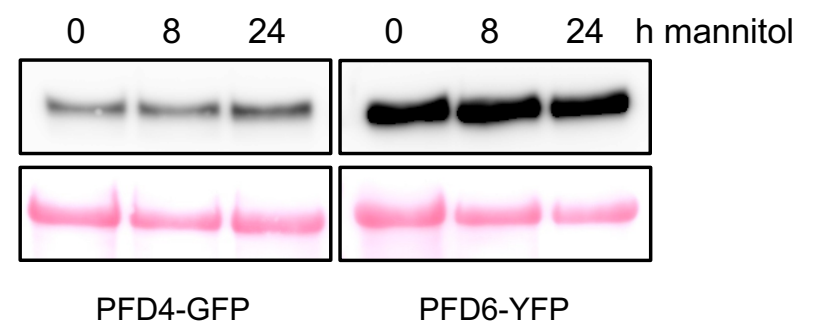

Figure S8 
(a)

(b)
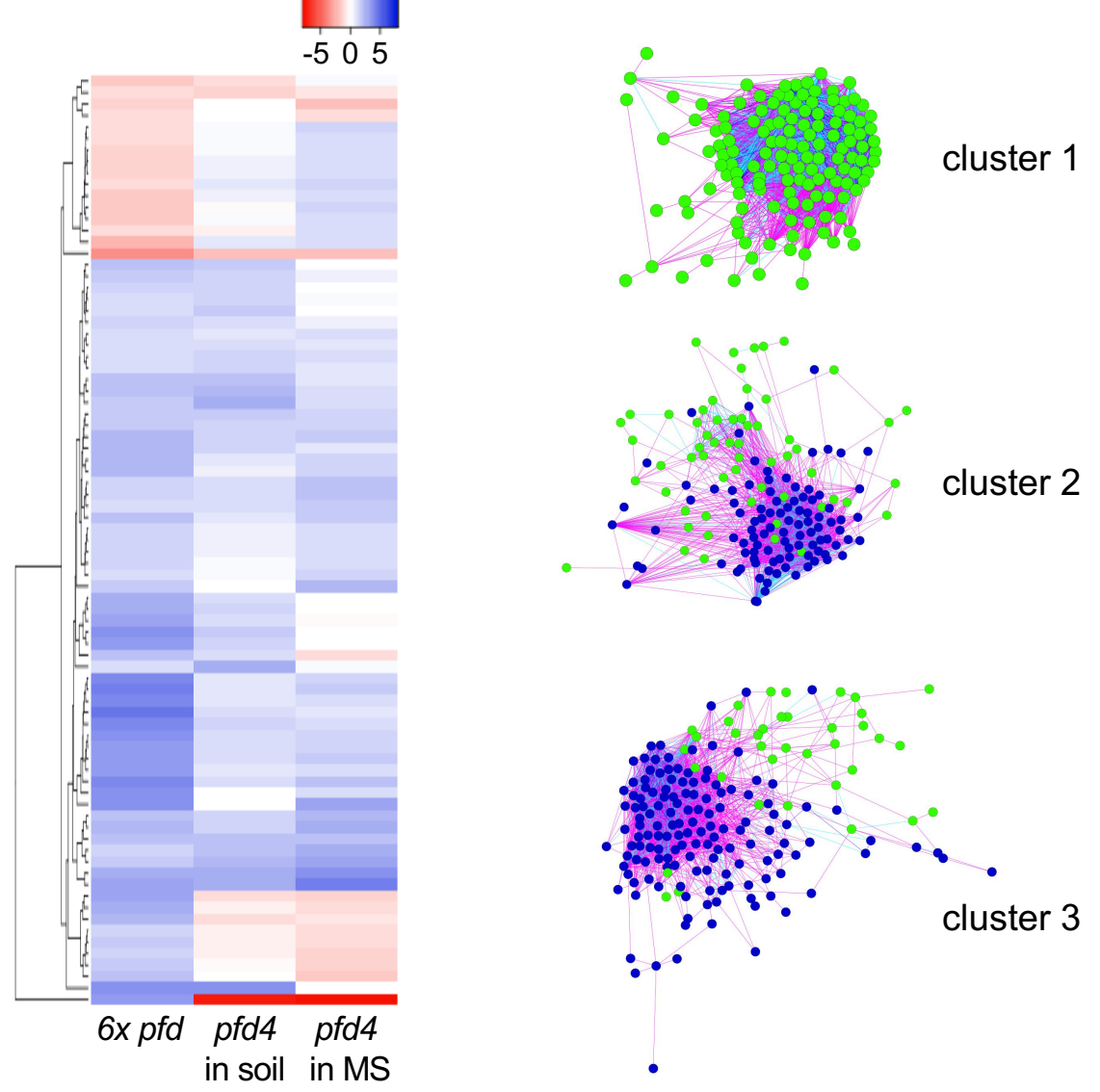



(c)

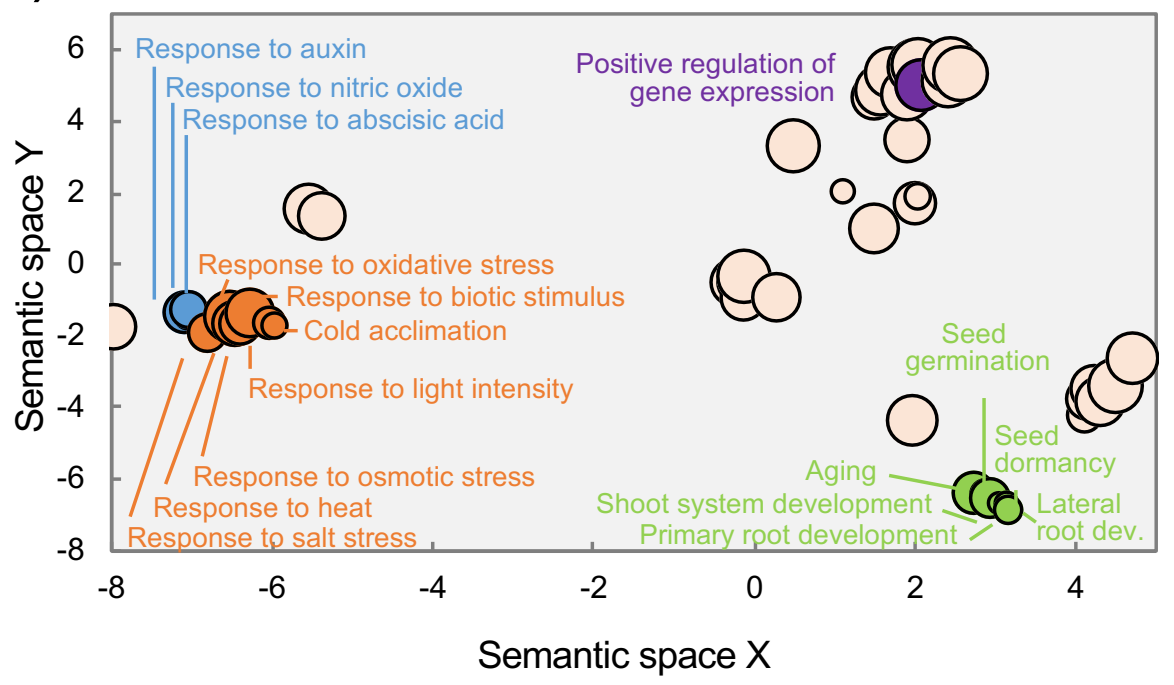

Figure S9 


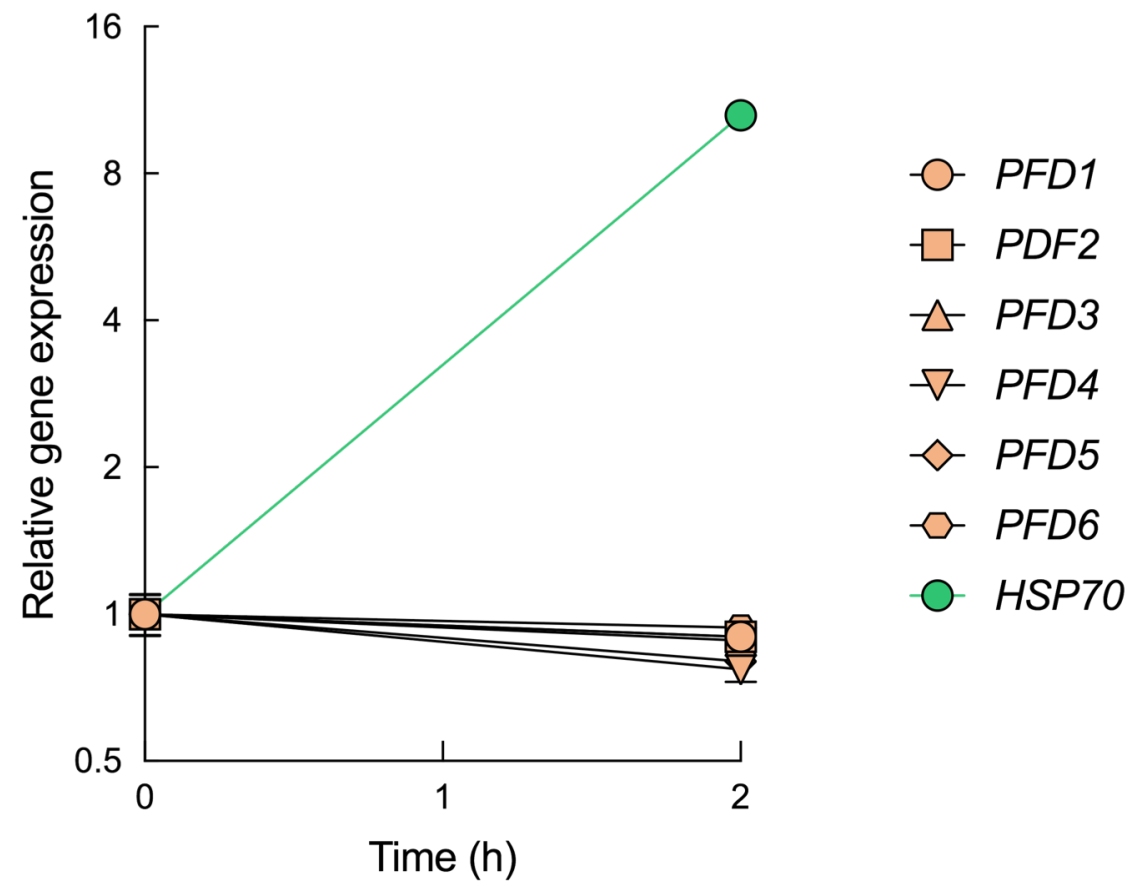

Figure S10 


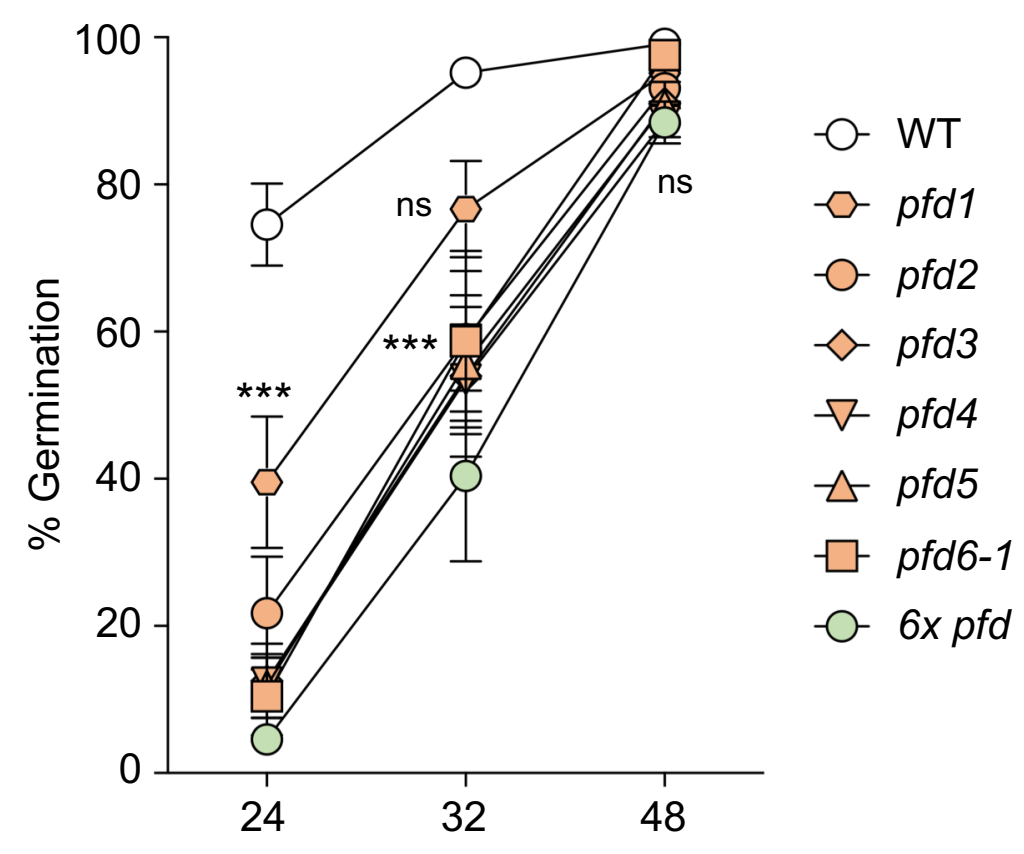

Hours after stratification

Figure S11 

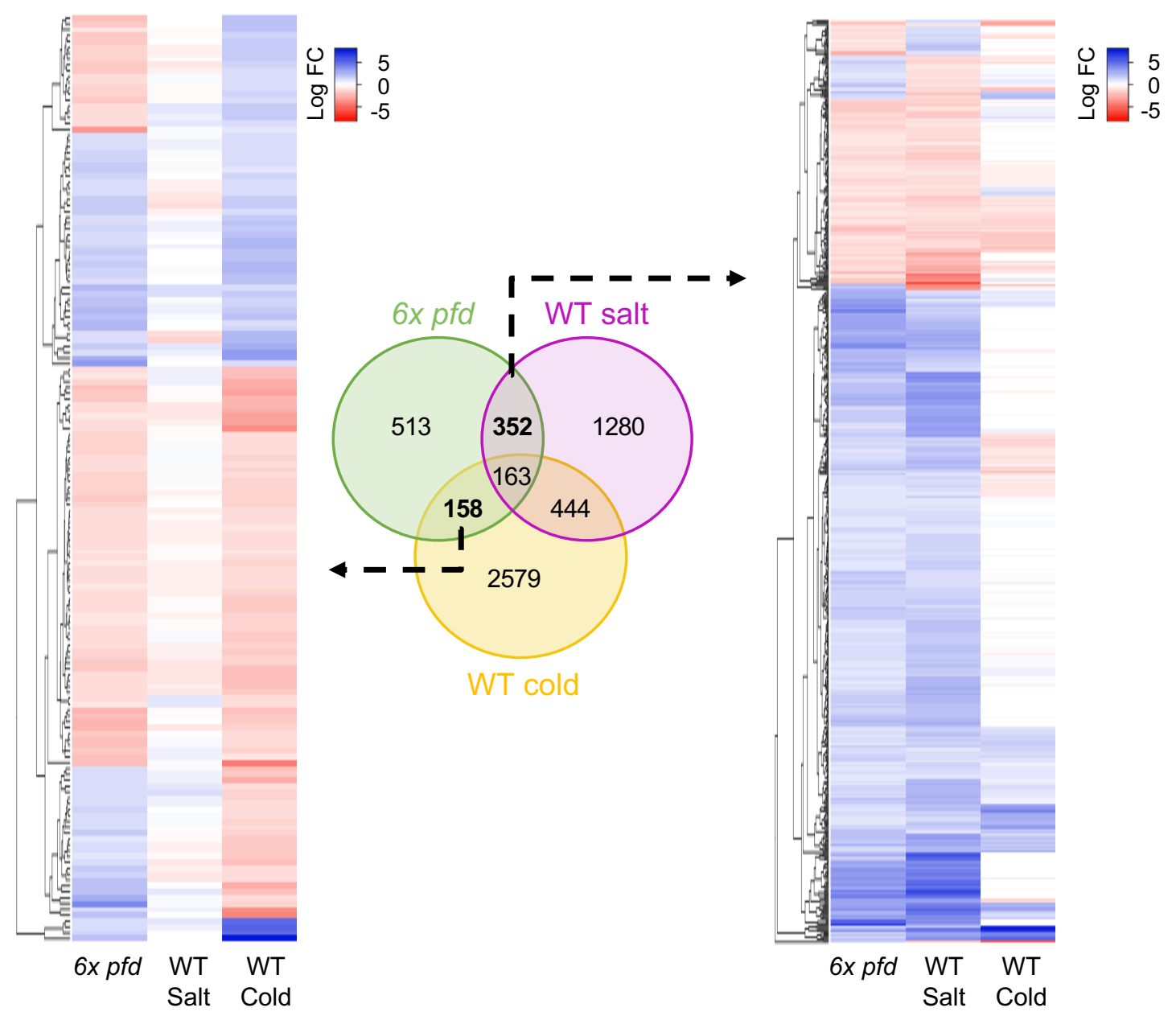

Figure S12 
(a)

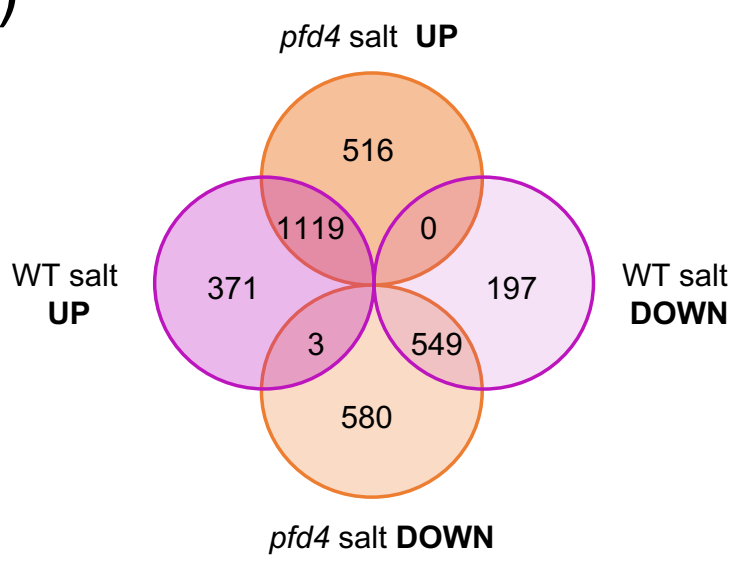

(b)

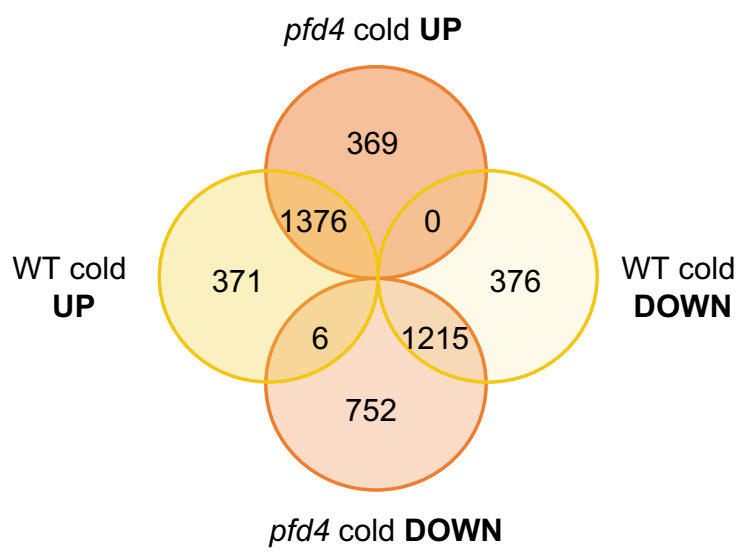

Figure S13 